\title{
Self Organization of Tilts in Relay Enhanced Networks: A Distributed Solution
}

\author{
Ali Imran ${ }^{1}$, Muhammad Ali Imran ${ }^{2}$, Adnan Abu-Dayya ${ }^{1}$ and Rahim Tafazolli ${ }^{2}$ \\ ${ }^{1}$ Qatar Mobility Innovation Center (QMIC), Doha, Qatar \\ ${ }^{2}$ CCSR, University of Surrey, Guildford, United Kingdom. GU2 7XH. \\ email: $\{\text { alii, adnan }\}^{1} @$ qmic.com; $\{\text { m.imran, r.tafazolli }\}^{2} @$ surrey.ac.uk
}

\begin{abstract}
Despite years of physical-layer research, capacity enhancement potential of relays is limited by the additional spectrum required for Base Stations (BS)-Relay Station (RS) links. This paper presents a novel distributed solution by exploiting a system level perspective instead. Building on a realistic system model with impromptu RS deployments, we develop an analytical framework for tilt optimization that can dynamically maximize spectral efficiency of both BS-RS and BS-user links in online manner. To obtain a distributed self-organizing solution, the large scale system-wide optimization problem is decomposed into local small scale subproblems by applying the design principles of self-organization in biological systems. The local subproblems are non-convex but having a very small scale can be easily solved via standard nonlinear optimization techniques such as sequential quadratic programming. The performance of developed solution is evaluated through extensive simulations for LTE-A type system and compared against number of benchmarks including a centralized solution obtained via brute force, that also gives an upper bound to assess optimality gap. Results show that proposed solution can enhance average spectral efficiency by up to $50 \%$ compared to fixed tilting with negligible signaling overheads. The key advantage of the proposed solution is its potential for autonomous and distributed implementation.
\end{abstract}

\section{Index Terms}

Self Organization; Tilt Optimization; Relay station; Spectral Efficiency Maximization

\section{INTRODUCTION}

Quest for higher data rates and better quality of service is pushing the wireless cellular systems to their physical limits [1]. More extensive use of Relay Stations (RS) have been identified as 
one of the key strategies to meet the unprecedented high demands in future cellular systems such as LTE-A. Compared to conventional Base Station (BS), RSs are generally cheaper, more energy efficient and quick roll-out friendly solution to extend coverage and capacity of cellular systems [2]. However, to exploit the full advantages of the RSs, e.g. as intended in LTE-A, two key problems remain challenging to date. The first major problem is to overcome the inherent drawback of the RS i.e. the spectrum reuse inefficiency caused by the extra spectrum required for BS-RS access link as illustrated in figure 1. The need for this extra spectrum severely limits RS' potential of system-wide capacity enhancement in cellular systems [3]. Therefore, it is very desirable to optimize the spectral efficiency of access links so that more spectrum is available for RS-user and BS-user coverage links.

Secondly, as identified by 3GPP [4], in future cellular networks such as LTE-A the BS infrastructure that has to support a RS based enhancement, should have Self Organization (SO) capabilities to accommodate the impromptu deployment of the RSs. Such on-the-run random deployment of RSs in time and space is envisioned to be inevitable to cope with spatio-temporally dynamic demands of coverage and capacity in future cellular systems. SO will be particularly required to accommodate advent or departure or location change of RSs. Without proper SO capabilities in BSs, the wide scale deployment of new RSs can be almost as demanding as deployment of new BSs, thereby severely limiting advantages of RSs.

The framework presented in this paper addresses both of these challenges simultaneously. i.e. 1) it enhances spectral efficiency on BS-RS access link (without compromising BS-user link spectral efficiency) and thus reduces spectrum reuse inefficiency caused by RSs access links; and 2) it ensures continuous maintenance of the optimal spectral efficiency through a distributed tilt SO solution for BSs to cope with the on-the-run deployment of RSs.

\section{A. Novelty and Contributions}

While exhaustive research efforts have been channeled to develop myriad of physical layer [5], MAC layer [6] and network layer [7] solutions to counter measure the spectrum reuse inefficiency caused by the access links of RSs, remarkably very less attention has been channelled to the solutions that can be harnessed with a system level perspective. In this paper by exploiting the system level perspective, we present a novel framework for spectral efficiency enhancement on the access link through distributed self-optimization of system-wide BS antenna tilts. 
Given the significance of tilt optimization in cellular system, a large number of works have already embarked on this problem in context of macro cellular systems $[8]-[26]^{1}$. In order to cope with NP-Hard nature of the problem, these works have mainly resorted to heuristics such as tabu-search [8], fuzzy reinforcement learning [15], fuzzy q-learning [23], golden section search [28], Taguchi method [20], multi-level random Taguchi's method [24], reinforcement learning based sparse sampling [25] and simulated annealing [26]. The general methodology followed in these works has been to evaluate the desired Key Performance Indicator(s) (KPIs) as a function of system-wide tilt angles through a simulation model. A non-exhaustive search is then carried out by exploration of the solution space in vicinities selected with help of one of the aforementioned heuristics for obtaining the suitable tilt values. Given the limited transparency of the simulation models that acts as a black box between tilt value and the KPI and inherent lack of guarantee from these heuristics that the solution produced is close to optimal, the quality of the solution yielded by this methodology remains hard to be asserted. Furthermore, the long time required to search an acceptable system-wide solution using this approach relying on sophisticated offline planning tools is another factor that thwarts the practical implementation of such solutions for self organising antenna tilts in online manner. Lack of repeatability and no convergence guarantee is another hurdle in use of this approach for self organization of tilts in live networks. To overcome these challenges, in this paper we exploit a mathematical framework to model the KPI of interest as a function of tilt, thereby obtaining a more transparent system model that allows deeper insights and thus better control of system behaviour. Then, instead solving for the system-wide NP-hard problem through a heuristic, we propose to decompose the problem into local sub-problems that because of being of very small scale, can be solved by more deterministic methods and thus can have better quality assurance.

Another novelty of our work is that prior works have mainly focused on tilt optimization in macro cellular systems and do not consider a relay enhanced cellular systems with consideration of BS-RS access links as we do in this work. Only in [29] authors have introduced the concept of spectral efficiency enhancement on access link through BS antenna tilt adaptation for the first time. However, the scope of [29] is limited to an ideally symmetric scenario where all the cells are assumed to contain strictly one RS in each cell. Thus it does not take into account more

\footnotetext{
${ }^{1}$ A detailed survey of works on tilt optimization can be found in our previous work in [27].
} 
generic scenarios of heterogeneous deployment where RSs are deployed quite randomly and some cells might not contain RSs and some users are directly served by BSs. The gain of the solution proposed in [29] is demonstrated with a model consisting of only three cells whereas we conduct performance evaluation using a full scale system model. Also, while considering BS-RS link, solution in [29] does not take into account the impact of BS antenna tilting on the BS-user links, as we do in this work. Therefore, to the best of our knowledge, this paper presents a novel distributed solution for run-time self optimization of system-wide tilts in relay enhanced cellular system with realistic heterogeneous deployments. We compare the performance of our proposed solution with three different benchmarks.1) Performance with no tilting in the system 2)Performance with a range of pragmatic fixed tilting values that are generally used in state of the art commercial cellular networks. This includes fixed optimal tilt values depending on cell size and antenna height 3) Performance with a globally optimal centralized SO solution obtained for a small scale version of the problem through brute force method. The key advantage of the proposed solution is that it can enhance average BS-RS as well as BS-user link spectral efficiency while dynamically coping with run time addition of relays in the system without requiring centralized signalling and manual reconfiguration of BSs antenna tilts.

\section{B. Organization of paper}

The rest of paper is organized as follows: in section II we present system model, assumptions and problem formulation. In order to achieve a SO solution, in section III we propose a way to decompose the system-wide problem into local subproblems as inspired by SO systems in nature. Solution methodology for local subproblems is also presented in this section. Section IV presents numerical as well as system level simulation results to demonstrate the gains achievable by the proposed solution. Pragmatic implementation of proposed solution in context of LTE-A is given in section $\mathrm{V}$ and section VI concludes this paper.

\section{System Model And Assumptions}

\section{A. Assumptions and Nomenclature}

The analysis in this paper focuses only on the down link of cellular systems for sake of conciseness. It is assumed that all user devices as well as RSs have omnidirectional antennas with a constant gain in all directions. The term sector is used in the same meanings as a cell. Frequency 


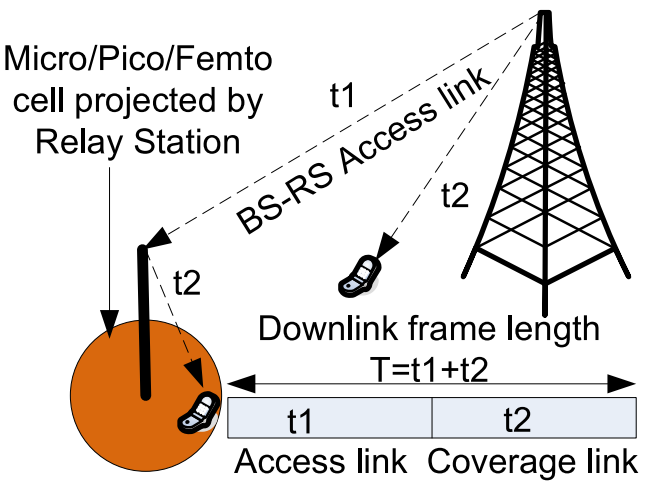

Fig. 1. The extra spectrum required for access link causes spectrum reuse inefficiency. This inefficiency can be decreased by maximising the spectral efficiency on access link as that will allow reduction in $\mathrm{t} 1$ and an increase in $\mathrm{t} 2$.

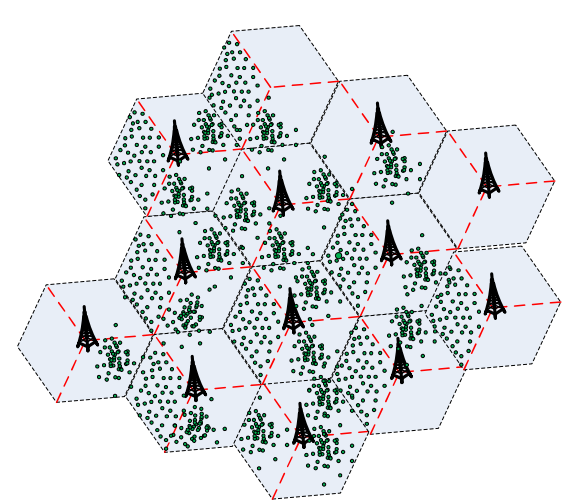

Fig. 2. System model for problem formulation. Small (red) circles show RS that are randomly located in some sectors to cover hotspots etc.

reuse of one is considered and consequently we assume an interference limited scenario where noize is negligible compared to interference. Since, the time scale of self optimization of tilts will be in the order of hours to days, therefore, short term channel variations i.e. fast fading are omitted in the analytical model for better tractability. However, the features omitted in the analysis namely noise and fast fading are modeled in detail in the simulation model used for performance evaluation in Section IV to assess the performance of proposed solution in more realistic scenarios. We use term throughput in this paper with similar meaning as the bandwidth normalized ergodic capacity given by $\log _{2}(1+S I R)$ that is thus equivalent to the spectral efficiency in $\mathrm{b} / \mathrm{s} / \mathrm{Hz}$, where SIR stands for Signal to Interference Ratio. BS and RS multiplex in time (or frequency) such that there is no interference between RS-user and BS-RS links as illustrated in figure 1. Due the geometrical context of the paper, while refereing to BS, RS and users we will be referring to the locations of their antennas until unless specified otherwise. Symbol tilde e.g. in $\tilde{x}$ is used to denote optimal value of a variable $x$ and symbol hat e.g. in $\hat{x}$ is used to denote an approximation of a variable $x$.

\section{B. System Model}

We consider a sectorized multi cellular network as shown in figure 2. Each BS has three cells and each cell has at most one RS station in it placed at arbitrary location. The purpose of RS can be to cover a random user hotspot for capacity enhancement or to fill a coverage whole for coverage enhancement. Let $\mathcal{B}$ denote the set of points corresponding to the transmission antenna location of all BS cells, $\mathcal{R}$ denote the set of points representing locations of the RSs antennas 


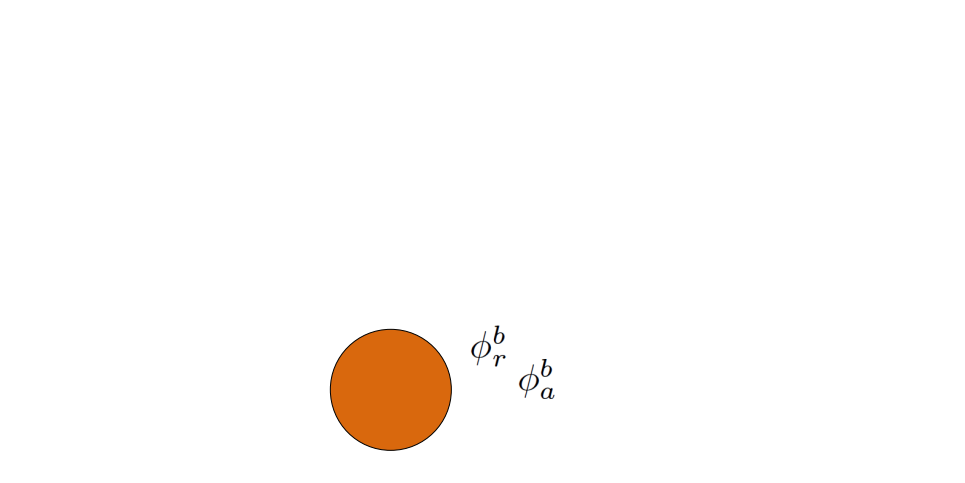

Fig. 3. Illustration of geometrical background of the analysis.

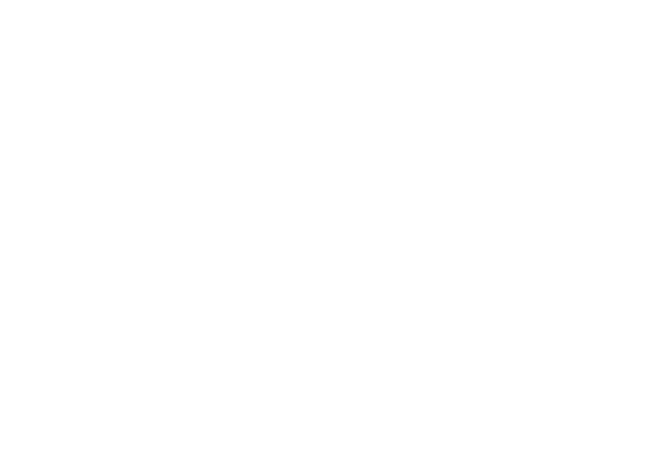

Fig. 4. The optimal tilt $\tilde{\psi}_{\text {tilt }}^{b}$ can be mapped to a locus of points equidistant distant from the BS.

in the system and $\mathcal{U}$ denote set of points representing all the user devices randomly located in the system. The geometric SIR on the access link of a RS located at point $r \in \mathcal{R}$ associated with $b^{\text {th }}$ BS cell, can be given as:

$$
\gamma_{r}^{b}=\frac{P^{b} G_{r}^{b} G_{r} \delta_{r}^{b} \alpha\left(d_{r}^{b}\right)^{-\beta}}{\sum_{\forall \dot{b} \in \mathcal{B} \backslash b}\left(P^{b} G_{r}^{b} G_{r} \delta_{r}^{\dot{b}} \alpha\left(d_{r}^{b}\right)^{-\beta}\right)} \quad b, \hat{b} \in \mathcal{B}, r \in \mathcal{R}
$$

where $P^{b}$ is transmission power of the $b^{\text {th }}$ BS cell, $d_{r}^{b}$ and $d_{r}^{b}$ are distances between the $b^{\text {th }}$ and $b^{\text {th }}$ BS cell (transmitting) antenna locations and (receiving) RS antenna location $r$. The $\alpha$ and $\beta$ are pathloss coefficient and exponents respectively that can be used to model a generic pathloss model. $\delta_{r}^{b}$ and $\delta_{r}^{b}$ are shadowing coefficients that represent shadowing faced by a signal at location $r$ while being received from the $b^{\text {th }}$ and $b^{\text {th }}$ BS antennas, respectively. Note that $\delta_{r}^{b}$ and $\delta_{r}^{b}$ are not assumed to be same, despite of being shadowing values at same location, because in order to model more realistic prorogation scenario we take into account the dependency of shadowing values on the angles of arrival, using the multi-cell cross-correlation shadowing model proposed in [30]. The operator ' $\backslash$ ' in $\mathcal{B} \backslash b$ means all elements of $\mathcal{B}$ except $b$.

$G_{r}^{b}$ and $G_{r}^{b}$ are antenna gains perceived at $r^{t h}$ RS from $b^{t h}$ and $b^{t h}$ BS respectively. For 3GPP LTE the three dimensional antenna pattern can be modelled as proposed in [31]. Using the geometry in figure 3 the perceived antenna gain from $b^{\text {th }} \mathrm{BS}$, at location $r$ of a RS can be written in as follows:

$$
G_{r}^{b}=100^{0.1}\left(\lambda_{v}\left(G_{\max }-\min \left(12\left(\frac{\psi_{r}^{b}-\psi_{\text {tilt }}^{b}}{B_{v}}\right)^{2}, A_{\max }\right)\right)+\lambda_{h}\left(G_{\max }-\min \left(12\left(\frac{\phi_{r}^{b}-\phi_{a}^{b}}{B_{h}}\right)^{2}, A_{\max }\right)\right)\right)
$$

where $\psi_{r}^{b}$ is the vertical angle at $b^{\text {th }}$ cell in degrees from reference axis (horizon) to the RS $r$. $\psi_{\text {tilt }}^{b}$ is the tilt angle of the $b^{\text {th }}$ cell as shown in figure 3. The $\phi_{a}^{b}$ is angle of the azimuth orientation of the antenna with respect to horizontal reference axis i.e. positive $\mathrm{x}$-axis. $\phi_{r}^{b}$ is the angle of 
location $r^{t h}$ RS from the horizontal reference axis, at $b^{t h}$ BS. Subscripts $h, a$ and $v$ denote horizontal, azimuth and vertical respectively. Thus $B_{h}$ and $B_{v}$ represent horizontal and vertical beamwidths of the BS antenna respectively, and $\lambda_{h}$ and $\lambda_{v}$ represent weighting factors for the horizontal and vertical beam pattern of the antenna in 3D antenna model [31] respectively. $G_{\max }$ and $A_{\max }$ denote the maximum antenna gain at the boresight of the antenna and maximum antenna attenuation at the sides and back of the boresight of the antenna respectively in $\mathrm{dB}$. $G_{\max }$ and $A_{\max }$ are same for horizontal and vertical radiation pattern, therefore, no subscript $v$ and $h$ are associated to them.

In order to substitute in (8), the antenna model can be simplified by neglecting the maximum attenuation factor $A_{\max }$ and assuming the maximum gain $G_{\max }$ as $0 \mathrm{~dB}$ in (2). Both of these assumptions preserve the accuracy of this antenna model essential to our analysis i.e. parabolic dependency of antenna gain on angular distance from the boresight stays unchanged. At the same time these assumptions allow the analytical tractability and insights that otherwise will not be possible. Nevertheless, these assumptions will be removed in the simulation and numerical analysis presented in Section IV, and therefore the results presented in this paper depict the performance of the proposed solution in a system model without these simplifications. The simplified antenna model can be written as:

$$
G_{r}^{b}=10^{-1.2\left(\lambda_{v}\left(\frac{\psi_{r}^{b}-\psi_{t i l t}^{b}}{B_{v}}\right)^{2}+\lambda_{h}\left(\frac{\phi_{r}^{b}-\phi_{a}^{b}}{B_{h}}\right)^{2}\right)}
$$

We assume that all the base stations transmit with same power and all RS antennas have constant gain in all directions i.e. $G_{r}=$ constant. Thus, by using (3) in (1) the SIR on the access link of the $r^{\text {th }} \mathrm{RS}$ can be determined as:

$$
\gamma_{r}^{b}=\frac{\delta_{r}^{b} \alpha\left(d_{r}^{b}\right)^{-\beta} 10^{-1.2\left(\lambda_{v}\left(\frac{\psi_{r}^{b}-\psi_{t i l t}^{b}}{B_{v}}\right)^{2}+\lambda_{h}\left(\frac{\phi_{r}^{b}-\phi_{a}^{r}}{B_{h}}\right)^{2}\right)}}{\sum_{\forall \hat{b} \in \mathcal{B} \backslash b}\left(\delta_{r}^{\hat{b}} \alpha\left(d_{r}^{\hat{b}}\right)^{-\beta} 10^{\left.-1.2\left(\lambda_{v}\left(\frac{\psi_{r}^{\hat{b}}-\psi_{t i l t}^{\hat{b}}}{B_{v}}\right)^{2}+\lambda_{h}\left(\frac{\phi_{r}^{b}-\phi_{a}^{b}}{B_{h}}\right)^{2}\right)\right)}\right.}
$$

For the ease of expression we use following substitutions:

$$
\begin{aligned}
& c_{k}^{b}=\frac{B_{v}^{2} \lambda_{h}}{\lambda_{v}}\left(\frac{\phi_{r}^{b}-\phi_{a}^{b}}{B_{h}}\right)^{2} ; \quad c_{k}^{b}=\frac{B_{v}^{2} \lambda_{h}}{\lambda_{v}}\left(\frac{\phi_{r}^{\hat{b}}-\phi_{a}^{\hat{b}}}{B_{h}}\right)^{2} \\
& h_{r}^{b}=\delta_{r}^{b} \alpha\left(d_{r}^{b}\right)^{-\beta} ; \quad h_{r}^{\hat{b}}=\delta_{r}^{\hat{b}} \alpha\left(d_{r}^{\hat{b}}\right)^{-\beta} ; \quad \mu=\frac{-1.2 \lambda_{v}}{B_{v}^{2}}
\end{aligned}
$$


Using the substitutions in (5)-(6), the SIR in (4) can be written as:

$$
\gamma_{r}^{b}=\frac{h_{r}^{b} 10^{\mu\left(\left(\psi_{r}^{b}-\psi_{t i l t}^{b}\right)^{2}+c_{r}^{b}\right)}}{\left.\sum_{\forall b \in \mathcal{B} \backslash \hat{b}}\left(h_{r}^{\hat{b}} 10^{\mu\left(\left(\psi_{r}^{\hat{b}}-\psi_{\text {tilt }}^{b}\right)^{2}+c_{r}^{b}\right.}\right)\right)}
$$

Note that $\gamma_{r}^{b}$ is function of vector of tilt angles of all sectors i.e. $\boldsymbol{\psi}_{\text {tilt }}^{B}=\left[\psi_{\text {tilt }}^{1}, \psi_{\text {tilt }}^{2}, \psi_{\text {tilt }}^{3} \ldots \psi_{\text {tilt }}^{B}\right]$ where $B=|\mathcal{B}|$, but for sake of simplicity of expression we will show this dependency only where necessary. Similarly the geometric SIR perceived by a user at a location $u$ being served by $b^{\text {th }}$ BS cell can be given as:

$$
\gamma_{u}^{b}=\frac{P^{b} G_{u}^{b} \alpha\left(d_{u}^{b}\right)^{-\beta}}{\sum_{\forall \dot{b} \in \mathcal{B} \backslash b}\left(P^{\dot{b}} G_{u}^{b} \alpha\left(d_{u}^{b}\right)^{-\beta}\right)} \quad b, \hat{b} \in \mathcal{B}, u \in \mathcal{U}
$$

where $d_{u}^{b}$ and $d_{u}^{b}$ are distances between the $b^{\text {th }}$ and $b^{t h}$ BS cell and $u^{\text {th }}$ user. Following same steps as above, the SIR for the BS-user link can be written as:

$$
\gamma_{u}^{b}=\frac{h_{u}^{b} 10^{\mu\left(\left(\psi_{u}^{b}-\psi_{\text {tilt }}^{b}\right)^{2}+c_{u}^{b}\right)}}{\sum_{\forall \dot{b} \in \mathcal{B} \backslash b}\left(h_{u}^{\dot{b}} 10^{\mu\left(\left(\psi_{u}^{\hat{b}}-\psi_{\text {tilt }}^{\hat{b}}\right)^{2}+c_{u}^{\hat{b}}\right)}\right)}
$$

\section{TILT OPTIMIZATION FRAMEWORK}

In this section, first the problem is formulated using the system model. Key steps to design a SO solution are identified by inspirations from natural SO systems. These steps are are then applied on our system model to design the analytical framework for a SO solution.

\section{A. Problem Formulation}

As our objective is to minimize the radio resources required for access link and thus maximize the net gain of RSs in terms of system-wide capacity. To achieve this objective, we propose to optimize system-wide BS antenna tilts such that it maximize the long term weighted average bandwidth normalized throughput $\eta$ i.e. weighted average spectral efficiency (bandwidth normalized ergodic capacity) on all the access links in the system. Mathematically our problem can be written as:

$$
\max _{\boldsymbol{\psi}_{\text {tilt }}^{B}} \eta\left(\boldsymbol{\psi}_{\text {tilt }}^{B}\right)=\max _{\boldsymbol{\psi}_{\text {tilt }}^{B}} \frac{1}{W_{r}} \sum_{\forall r \in \mathcal{R}} w_{r} \log _{2}\left(1+\gamma_{r}^{b}\left(\boldsymbol{\psi}_{\text {tilt }}^{B}\right)\right)
$$

where $0<w_{r} \leq 1$ is a weight factor that varies over a fixed range of $0-1$ and can be assigned to each RS to reflect the relative importance of its backhaul link in overall system level optimization. 
In other words, these weights can be set to model the significance of each RS depending on statistics of numbers and activity levels of users it serves. Thus these weights can also be used to reflect if a RS has been deployed for coverage extension and therefore might have low load backhaul that need to be assigned lower weight, or if the RS is for capacity extension at a hotspot and therefore might have heavily loaded backhaul and that need to be assigned proportionally higher $w_{r}$. Where, $W_{r}=\sum_{\forall r \in \mathcal{R}} w_{r}$. In a simple example, $w_{r}$ can be calculated as follows:

$$
w_{r}=\frac{\sum_{\forall u \in \mathcal{U}_{b}^{r}} a_{u}}{\sum_{\forall u \in \mathcal{U}_{b}} a_{u}} \quad, 0<a_{u} \leq 1
$$

Where $a_{u}$ represents $u^{\text {th }}$ user activity level. $\mathcal{U}_{b}$ is set of users in $b^{\text {th }}$ BS cell and $\mathcal{U}_{b}^{r}$ is set of users in the $r^{t h}$ RS cell within $b^{t h}$ BS cell. However, adapting BS antenna tilt will have an impact on the users that are directly served by BS. To take these users into account the problem in (10) can be reformulated as:

$$
\max _{\boldsymbol{\psi}_{\text {tilt }}^{B}}\left(\frac{1}{W_{r}} \sum_{\forall r \in \mathcal{R}} w_{r} \log _{2}\left(1+\gamma_{r}^{b}\left(\boldsymbol{\psi}_{\text {tilt }}^{B}\right)\right)+\frac{1}{A_{u}} \sum_{\forall u \in \mathcal{U} \backslash \mathcal{U}} a_{u} \log _{2}\left(1+\gamma_{u}^{b}\left(\boldsymbol{\psi}_{\text {tilt }}^{B}\right)\right)\right)
$$

Where $\mathcal{U}$ is set of users served by RSs such that $\mathcal{U} \subset \mathcal{U}$ and thus users served directly by BS are given by set $\mathcal{U} \backslash \mathcal{U}$ and $\hat{A}_{u}=\sum_{\forall u \in \mathcal{U} \backslash \dot{\mathcal{U}}} a_{u}$.

The formulation in (12) is a nonlinear multi variable optimization problem. Its solution would require global cooperation among all cells in the system and hence can not be implemented as a distributed SO solution [27], [32]. Furthermore, as we will see in subsection III-E the objective function in (12) is non-convex. Also huge number of the optimization variables i.e. $\boldsymbol{\psi}_{\text {tilt }}^{B}=\left[\psi_{\text {tilt }}^{1}, \psi_{\text {tilt }}^{2}, \psi_{\text {tilt }}^{3} \ldots \psi_{\text {tilt }}^{B}\right]$ means it is a large scale optimization problem. Therefore, the conventional heuristic based approach of finding a sub-optimal solution by using an offline planning tools, do not offer a pragmatic mechanism for online self optimization of tilts due to the very large computational time. Furthermore, lack of guarantee for quality of solution and limitations of the off line planning tool to depict live network may not only compromise the agility of a closed loop nature of an ideal SO solution but also may increase instability risks in SO process [27]. In following section we present a novel biologically inspired mathematical framework that can enable self optimization of tilts by providing a distributed solution of (12). 


\section{B. Designing a Self Organising Solution}

In nature many systems can be observed to exhibit self organizing behaviour. A detailed discussion on designing self organization by mimicking such systems can be found in our works in [27], [33] as well as in [32], [34], [35]. Here it would suffice to say that, for a distributed self organising solution, perfect objective may not be aimed for at system-wide level [32]. Rather, an approximation of the objective can be aimed for, given that it can be decomposed into subobjectives that can be achieved at local level while requiring interactions only among local entities of system. This phenomenon, in turn can approximately achieve the original system wide objective resulting into emergence of self organising behaviour [27], [32].

This design principle of SO when applied to our problem in (12) means, given the complexity of this problem, we need to 1) find an alternative approximate manifestation of the problem in (12) that can be then 2) decomposed down into easily solvable local sub-problems whose solution would at most require local coordination only among neighbouring cells. And finally we need to 3) determine the solution of those local subproblems. In following three subsections we follow these three steps to achieve a distributed self organising solution for problem in (12).

\section{Simplifying the Problem to Achieve Decomposability}

Difficulty to obtain pragmatic solution of (12) stems mainly from fact there is summation in the optimization objective that grows with number of users and complexity of each term in the summation also grows with number of cells in the system. In the sequel we present the analysis to determine a significantly simpler and scalable manifestation of (12) as desired for the distributed SO solution.

Theorem 1. If a cell has uniform user distribution and the importance of each geographical point $(x, y)$ in the cell is given by weight $a_{(x, y)}$, the antenna tilt $\tilde{\psi}_{\text {tilt }}^{b}$ of that cell is optimal in terms of weighted average area spectral efficiency if it satisfies following condition:

$$
\int_{x} \int_{y} a_{(x, y)}\left(\left(\psi_{x, y}^{b}-\tilde{\psi}_{t i l t}^{b}\right) \frac{\tilde{\gamma}_{x, y}^{b}}{1+\tilde{\gamma}_{x, y}^{b}}\right) d x d y=0
$$

where $\tilde{\gamma}_{x, y}^{b}$ is the SIR perceived at point $(x, y)$ in cell $b$, when its antenna is tilted by $\tilde{\psi}_{\text {tilt }}^{b}$ degrees. $\tilde{\gamma}_{x, y}^{b}$ can be given as: 


$$
\tilde{\gamma}_{x, y}^{b}=\frac{d_{x, y}^{b}{ }^{-\beta} 10-1.2\left(\lambda_{v}\left(\frac{\psi_{x, y}^{b}-\tilde{\psi}_{\text {tilt }}^{b}}{B_{v}}\right)^{2}+\lambda_{h}\left(\frac{\phi_{x, y}^{b}-\phi_{t i l t}^{b}}{B_{h}}\right)^{2}\right)}{\sum_{\forall \dot{b} \in \mathcal{B} \backslash b}\left(d_{x, y}^{b}{ }^{-\beta} 10^{-1.2\left(\lambda_{v}\left(\frac{\psi_{x, y}^{b}-\psi_{t i l t}^{b}}{B_{v}}\right)^{2}+\lambda_{h}\left(\frac{\phi_{x, y}^{b}-\phi_{\text {tilt }}^{b}}{B_{h}}\right)^{2}\right)}\right)}
$$

Integral in (13) is surface integral over whole area of the cell projected by BS antenna at location $b$ and $(x, y)$ denote the coordinate of an arbitrary point in that cell.

Proof: Proof of theorem 1 is provided in Appendix A

Following corollaries can be deduced from theorem 1:

Corollary 1. If the tilt value for a given cell satisfies the condition:

$$
\sum_{u=1}^{\left|\mathcal{U}_{b}\right|} a_{u}\left(\left(\psi_{u}^{b}-\tilde{\psi}_{\text {tilt }}^{b}\right) \frac{\tilde{\gamma}_{u}^{b}}{1+\tilde{\gamma}_{u}^{b}}\right)=0
$$

it will yield greater or equal weighted average spectral efficiency on BS-user links than that obtained with any other value of tilt, for the same tilt angles of neighbouring cells. Mathematically

$$
\frac{1}{A_{u}^{b}} \sum_{\forall u \in \mathcal{U}_{b}} a_{u} \log _{2}\left(1+\gamma_{u}^{b}\left(\tilde{\psi}_{\text {tilt }}^{b}\right)\right) \geqslant \frac{1}{A_{u}^{b}} \sum_{\forall u \in \mathcal{U}_{b}} a_{u} \log _{2}\left(1+\gamma_{u}^{b}\left(\psi_{\text {tilt }}^{b}\right)\right) \quad \forall \quad 0 \leqslant \psi_{\text {tilt }}^{b} \leqslant 90
$$

where $\mathcal{U}_{b}$ is set of users in the $b^{\text {th }}$ cell and $A_{u}^{b}=\sum_{\forall u \in \mathcal{U}_{b}} a_{u}$. Note that $\gamma_{u}^{b}$ here is function of antenna tilt of the $b^{\text {th }}$ cell only, as rest of the antenna tilts are fixed.

Proof: Proof of corollary 1 directly follows proof of theorem 1 when generalised for a arbitrary user distribution wether uniform or non-uniform; and arbitrary user activity levels wether homogeneous or non-homogenous. (see result (56) in Appendix A)

Corollary 2. If $H^{b}$ and $H^{p}$ are heights of $b^{\text {th }}$ cell antenna and point $p$ and $d(p \leftrightarrow b)$ denotes distance between the $b^{\text {th }} B S$ and a user at point $p$; then the optimal tilt angle in that cell $\tilde{\psi}_{\text {tilt }}^{b}$ is the tilt that optimizes spectral efficiency at the point $p$ that belongs to a set of points $\mathcal{P}^{b}$ such that $\mathcal{P}^{b}=\left\{p \mid, d(p \leftrightarrow b)=d^{b}\right\}$ and $d^{b}=\left(H^{b}-H^{p}\right) / \tan \left(\tilde{\psi}_{\text {tilt }}^{b}\right)$.

Proof: This corollary follows theorem 1 through the fact that the optimal tilt angle $\tilde{\psi}_{\text {tilt }}^{b}$ given by theorem 1 can be transformed into a locus of points $\mathcal{P}^{b}$ that lie at distance $d^{b}$ from the $b^{\text {th }}$ cell antenna. This is illustrated in figure 4. 
Note that according to theorem 1 and its subsequent corollaries, a tilt angle of $b^{\text {th }}$ cell optimized for any of the points in set $\mathcal{P}^{b}$, optimizes average spectral efficiency in that cell. In other words set $\mathcal{P}^{b}$ represents set of focal points with respect to which tilt should be maximized in given cell with given user distribution and given user activity levels. However, in order to consider impact of interference from neighbouring cells and jointly optimize tilts, a single focal point in $\mathcal{P}^{b}$ need to be identified that can represent the user distribution in $b^{\text {th }}$ cell. Note that theorem 1 and subsequent corollaries have effectively reduced the search space for this single point from the whole cell area to a small set points given by $\mathcal{P}^{b}$. Now for any given user distribution in a cell this single focal point $p^{b} \in \mathcal{P}^{b}$ can be determined by invoking the classic definition of centre of gravity of a two dimensional mass distribution with an additional simplification that CG lies within $\mathcal{P}^{b}$, as follows:

$$
\tilde{p}^{b}=\arg \min _{\mathcal{P}^{b}} \sum_{\forall u \in \mathcal{U}_{b}} a_{u} d_{u}^{p}\left(p^{b} \leftrightarrow u\right)
$$

For ease of discussion we refer to this focal point as Centers of Gravity (CG) of a cell for its given user distribution and user activity profile. Fortunately, as long as users distribution and activity can be assumed uniform across the cell, CG can be shown to lie at the centroid of the trapezoid that constitutes the sector. In such case CG be determined based on cell size and antenna heights without having to take into account user distribution and activity profile.

Using theorem 1 and its corollaries, the users distribution in each cell can be represented by a single focal point for tilt optimization process for an arbitrary user distribution and user activity profile. If the collection of all such points in the system is given by the set $\mathcal{V}$ it can be defined as $\mathcal{V}=\bigcup_{\forall b \in \mathcal{B}} \tilde{p}^{b}$ where $\tilde{p}^{b} \in \mathcal{P}^{b}$. By using definition of $\mathcal{V}$ in conjunction with corollary 1 and 2 , the $2^{\text {nd }}$ summation of the right hand side of optimization problem in (12) can be written as:

$$
\frac{1}{\bar{A}_{u}} \sum_{\forall u \in \mathcal{U} \backslash \dot{\mathcal{U}}} a_{u} \log _{2}\left(1+\gamma_{u}^{b}\left(\boldsymbol{\psi}_{\text {tilt }}^{B}\right)\right) \equiv \sum_{\forall v \in \mathcal{V}} \log _{2}\left(1+\gamma_{v}^{b}\left(\boldsymbol{\psi}_{\text {tilt }}^{B}\right)+\sum_{\forall v \in \mathcal{V}} \log _{2}\left(1+\gamma_{v}^{b}\left(\boldsymbol{\psi}_{\text {tilt }}^{B}\right)\right)\right.
$$

Where $\mathcal{V}$ and $\check{\mathcal{V}}$ are sets of CGs representing BS associated users in cells with RS and without RS respectively, such that $\mathcal{V}=\{\dot{\mathcal{V}} \cup \check{\mathcal{V}}\}$. Substituting (18) in (12), the optimization problem can be written as:

$$
\max _{\boldsymbol{\psi}_{\text {tilt }}^{B}}\left(\frac{1}{W_{r}} \sum_{\forall r \in \mathcal{R}} w_{r} \log _{2}\left(1+\gamma_{r}^{b}\left(\boldsymbol{\psi}_{\text {tilt }}^{B}\right)\right)+\sum_{\forall \forall \in \mathcal{v}} \log _{2}\left(1+\gamma_{v}^{b}\left(\boldsymbol{\psi}_{\text {tilt }}^{B}\right)+\sum_{\forall \check{v} \in \mathcal{V}} \log _{2}\left(1+\gamma_{v}^{b}\left(\boldsymbol{\psi}_{\text {tilt }}^{B}\right)\right)\right)\right.
$$

To further simplify our optimization problem in (19) we propose following generic method to 


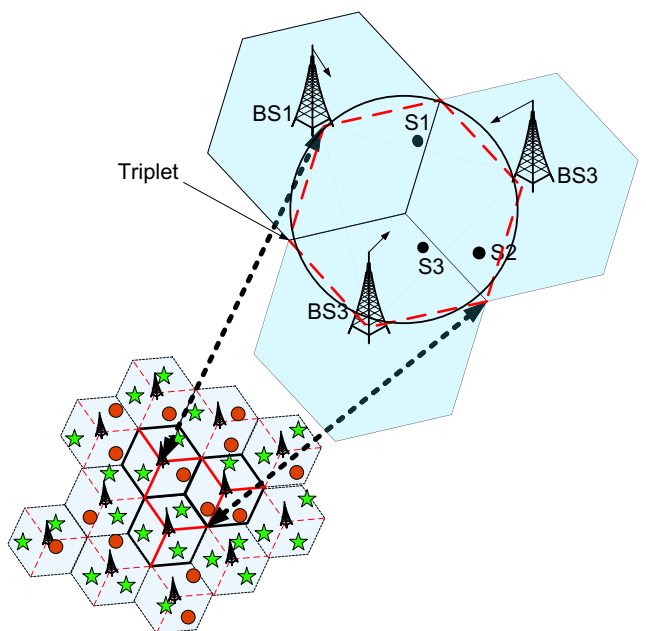

Fig. 5. Circles represent points in set $\mathcal{R}$ i.e. RS locations and stars represent points in set $\mathcal{V}$ i.e. focal points of user distributions in a cell determined through theorem (1) and its corollaries. Stars and circles together make set $\mathcal{S}$

determine a single point $s_{b}$ that can represent effective CG in each cell for the purpose of tilt optimization, including the cells that contain coverage or capacity enhancing RSs:

$$
s_{b}=\left\{\begin{array}{lll}
r_{b}, & \text { if }\left|\mathcal{U}_{b}^{r}\right|>0 \quad \& \quad & w_{r} \geq \frac{\sum_{\forall u \in \mathcal{U}_{b}^{b}} a_{u}}{\sum_{\forall u \in \mathcal{U}_{b}} a_{u}}, \text { where } r_{b} \in \mathcal{R} \\
\dot{v}_{b}, & \text { if }\left|\mathcal{U}_{b}^{r}\right|>0 \quad \& \quad w_{r}<\frac{\sum_{\forall u \in \mathcal{U}_{b}^{b}} a_{u}}{\sum_{\forall u \in \mathcal{U}_{b}} a_{u}}, \text { where } \dot{v} \in \mathcal{V} \\
\check{v}_{b}, & \text { otherwise, where } \check{v} \in \check{\mathcal{V}}
\end{array}\right.
$$

where $\mathcal{U}_{b}^{b}$ denotes set of users in $b^{\text {th }}$ cell that are directly associated with BS. Thus case 1 of (20) refers to the scenario where RS is serving majority of users and thus is expected has capacity limited backhaul link that must be considered in the tilt optimization process. This case is applicable to capacity enhancing RS installed at hotspots in a cell. The second case of (20) represents the cells where the main purpose of RS is coverage extension. The backhaul of such RS is not expected to be capacity limited and therefore does not have to be considered directly in tilt optimization problem. In this case the CG of the respective cell will be determined by the users associated directly with BS. Third case of equation (20) represents the cells with no RSs. Now if define $\mathcal{S}$ as set of all points $s_{b}$ in the system such that $|\mathcal{S}|=|\mathcal{B}|$, based on arguments presented above through (13)-(20), the problem in (19) can be written as:

$$
\max _{\boldsymbol{\psi}_{\text {tilt }}^{B}} \zeta\left(\boldsymbol{\psi}_{\text {tilt }}^{B}\right)=\max _{\boldsymbol{\psi}_{\text {tilt }}^{B}} \sum_{\forall s \in \mathcal{S}} \log _{2}\left(1+\gamma_{s}^{b}\left(\boldsymbol{\psi}_{\text {tilt }}^{B}\right)\right)
$$

The points (CGs) in set $\mathcal{S}$ are shown in figure 5, where circles represent RSs i.e. points in set $\mathcal{R}$; and stars represent the CGs of users' geographical distribution in cells with no RS or with 
RS whose backhaul is not critical for optimization process i.e. RS with $w_{r}<\frac{\sum_{\forall u \in \mathcal{U}_{b}^{b}} a_{u}}{\sum_{\forall u \in \mathcal{U}_{b}} a_{u}}$. Note that $|\mathcal{S}|<<|\mathcal{U} \backslash \mathcal{U} \cup \mathcal{R}|$. Thus, as highlighted in section III-B, for designing a SO solution, (21) is the required simplified manifestation of the original problem in (12).

\section{Decomposing the Simplified Problem into Local Subproblems}

As discussed in section III-B for a distributed SO solution, after simplifying the original problem in (12) into (21) its decomposition into local subproblems is required to transform it from a large scale optimization problem to a scale optimization problem. Such decomposition into local subproblems is common in SO systems in nature as explained via a case study of flock of common cranes above (see [36] and [33] for details). We refer to same case study and deduce the fact that, for achieving flock-wide objective of flying in V-formation, each crane merely relies on observation of its immediate two neighbours one on each side. Thus, although cranes do not achieve and maintain perfect $\mathrm{V}$ formation, they can still achieve up to $70 \%$ gain in group flight efficiency [36]. To exploit the same principle in our problem, we compromise slightly on global optimization of problem and propose a concept of triplet to enable its local decomposition. A triplet consist of three immediate neighbour cells that is illustrated in enlarged part of figure 5 and its use is explained and justified by following rather intuitive arguments:

Lemma 1. The average spectral efficiency at the CG's in the system when interference from only two immediate neighbouring sectors is considered, will be greater or equal to the average spectral efficiency at the same points when interference from all the sectors is considered. Mathematically $\hat{\zeta} \geqslant \zeta$ : where

$$
\hat{\zeta}=\frac{1}{|\mathcal{S}|} \sum_{\forall s \in \mathcal{S}} \log _{2}\left(1+\hat{\gamma}_{s}^{b}\left(\boldsymbol{\psi}_{\text {tilt }}^{\hat{B}}\right)\right)
$$

and

$$
\hat{\gamma}_{s}^{b}\left(\boldsymbol{\psi}_{\text {tilt }}^{\hat{B}}\right)=\frac{h_{s}^{b} 10^{\mu\left(\left(\psi_{s}^{b}-\psi_{\text {tilt }}^{b}\right)^{2}+c_{s}^{b}\right)}}{\sum_{\forall \check{b} \in \check{\mathcal{B}} \backslash b}\left(h_{s}^{\check{b}} 10^{\mu\left(\left(\psi_{s}^{\breve{b}}-\psi_{\text {tilt }}^{\breve{b}}\right)^{2}+c_{s}^{\breve{b}}\right)}\right)} \quad b, \check{b} \in \hat{\mathcal{B}}, \hat{\mathcal{B}} \subset \mathcal{B}
$$

$b$ here represents antenna location of arbitrary cell in which point $s$ lies and $\hat{\mathcal{B}}$ is set of $b^{\text {th }}$ and the two other most interfering cells adjacent to $b^{\text {th }}$ sector all mutually facing each other such that $|\hat{\mathcal{B}}|=\hat{B}=3$. The set of three cells represented by $\hat{\mathcal{B}}$ are termed as triplet as illustrated in figure 5 by dashed red lines. $\boldsymbol{\psi}_{\text {tilt }}^{\hat{B}}$ is vector of tilt angles of $\hat{B}$ sectors within the triplet. 
Proof: Lemma 1 is quite intuitive and in order to prove it we actually need to show that

$$
\begin{aligned}
& \gamma_{s}^{b}\left(\boldsymbol{\psi}_{\text {tilt }}^{B}\right) \leqslant \hat{\gamma}_{s}^{b}\left(\boldsymbol{\psi}_{\text {tilt }}^{\hat{B}}\right), \quad \forall s \in \mathcal{S}
\end{aligned}
$$

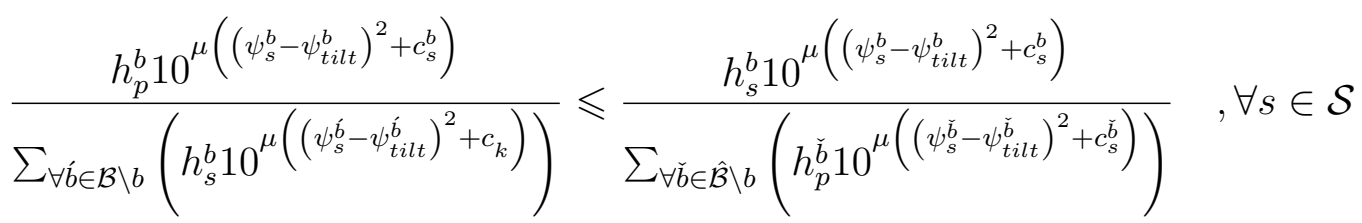

Multiplying both sides by inverse of the numerator and then inverting the both sides

$$
\sum_{\forall \dot{b} \in \mathcal{B} \backslash b}\left(h_{s}^{\dot{b}} 10^{\mu}\left(\left(\psi_{s}^{\dot{b}}-\psi_{t i l t}^{\dot{b}}\right)^{2}+c_{s}^{\dot{b}}\right)\right) \geqslant \sum_{\forall \check{b} \in \hat{\mathcal{B}} \backslash b}\left(h_{s}^{\check{b}} 10^{\mu\left(\left(\psi_{s}^{\check{b}}-\psi_{t i l t}^{\check{b}}\right)^{2}+c_{s}^{\check{b}}\right)}\right), \forall s \in \mathcal{S}
$$

By opening the left hand side

$$
\begin{aligned}
& \sum_{\forall \check{b} \in \check{\mathcal{B}} \backslash b}\left(h_{s}^{\check{b}} 10^{\mu\left(\left(\psi_{s}^{\check{b}}-\psi_{t i l t}^{\hat{b}}\right)^{2}+c_{s}^{\check{b}}\right)}\right)+\sum_{\forall \dot{b} \in B \backslash \hat{\mathcal{B}}}\left(h_{s}^{\dot{b}} 10^{\mu}\left(\left(\psi_{s}^{\dot{b}}-\psi_{t i l t}^{\dot{b}}\right)^{2}+c_{s}^{\dot{b}}\right)\right) \geqslant \\
& \sum_{\forall \check{b} \in \hat{\mathcal{B}} \backslash b}\left(h_{s}^{\check{b}} 10^{\mu}\left(\left(\psi_{s}^{\check{b}}-\psi_{t i l t}^{\check{b}}\right)^{2}+c_{s}^{\check{b}}\right)\right) \\
& \text { since } \sum_{\forall \dot{b} \in B \backslash \hat{\mathcal{B}}}\left(h_{s}^{\hat{b}} 10^{\mu}\left(\left(\psi_{s}^{\hat{b}}-\psi_{\text {tilt }}^{\dot{b}}\right)^{2}+c_{s}^{\hat{b}}\right)\right) \geqslant 0 \text { Hence the proposition in (24) is true. Since } \zeta \text { is }
\end{aligned}
$$
monotonically increasing function of $\gamma$, hence, $\gamma_{s}^{b} \leqslant \hat{\gamma}_{s}^{b}, \forall s \in \mathcal{S}$ implies that $\hat{\zeta} \geqslant \zeta$.

Corollary 3. As $\beta$ and the cell radius grows large, $\hat{\zeta}$ becomes closer approximation of $\zeta$

Proof: Corollary 3 can be easily proved by putting large values of $\beta$ and $d$ in (25).

Proposition 1. If the SIR is given by $\hat{\gamma}_{s}^{b}$, the maximum aggregate throughput achieved in the system by optimizing the tilts within each triplet independently, is same as the throughput achieved by optimizing system-wide tilts. Mathematically, $\hat{\zeta}_{N, \max }=\hat{\zeta}_{\max }$, where

$$
\hat{\zeta}_{\max }=\max _{\boldsymbol{\psi}_{\text {tilt }}^{B}} \hat{\zeta}\left(\boldsymbol{\psi}_{\text {tilt }}^{B}\right)=\max _{\boldsymbol{\psi}_{\text {tilt }}^{B} \forall s \in \mathcal{S}} \sum_{\forall} \log _{2}\left(1+\hat{\gamma}_{s}^{b}\right)
$$

where $\hat{\gamma}_{s}^{b}$ is the approximate SIR at point s given by (23) and

$$
\hat{\zeta}_{N, \max }=\sum_{\forall n \in \mathcal{N}} \hat{\zeta}_{n, \max }
$$

where

$$
\hat{\zeta}_{n, \text { max }}=\max _{\boldsymbol{\psi}_{\text {tilt }}^{T_{n}}} \sum_{\forall s \in \mathcal{S}_{n}} \log _{2}\left(1+\hat{\gamma}_{s}^{b}\left(\boldsymbol{\psi}_{\text {tilt }}^{T n}\right)\right), \quad \mathcal{S}_{n} \subset \mathcal{S}, \mathcal{T}_{n} \subset \mathcal{N}, \forall n \in \mathcal{N}
$$

where $\mathcal{T}_{n}$ is the $n^{\text {th }}$ triplet as illustrated in figure 5 and $\left|\mathcal{S}_{n}\right|=\left|\mathcal{T}_{n}\right|=T_{n}=3, \forall n \in \mathcal{N}, \boldsymbol{\psi}_{\text {tilt }}^{\boldsymbol{T}_{n}}$ is vector of tilt angles of sectors within $n^{\text {th }}$ triplet such that

$$
\mathcal{S}_{n} \cap \mathcal{S}_{n^{\prime}}=\Phi \quad \text { and } \quad \mathcal{T}_{n} \cap \mathcal{T}_{n^{\prime}}=\Phi \quad, \forall n \neq n^{\prime} \quad \text { where } n, n^{\prime} \in \mathcal{N}
$$


$\mathcal{N}$ is set of all such triplets, such that $|\mathcal{N}|=\frac{|\mathcal{B}|}{\left|\mathcal{T}_{n}\right|}$ is the total number of triplets in the system.

Proof: Since $|\mathcal{N}| \times\left|\mathcal{T}_{n}\right|=|\mathcal{N}| \times\left|\mathcal{S}_{n}\right|=|\mathcal{B}|=|\mathcal{S}|$ so (27) can be written as:

$$
\begin{aligned}
\hat{\zeta}_{\text {max }}=\max _{\boldsymbol{\psi}_{\text {tilt }}^{B}\{}\left\{\sum_{\forall s \in \mathcal{S}_{1}} \log _{2}\left(1+\hat{\gamma}_{s}^{b}\left(\boldsymbol{\psi}_{\text {tilt }}^{T_{1}}\right)\right)+\sum_{\forall s \in \mathcal{S}_{2}} \log _{2}\left(1+\hat{\gamma}_{s}^{b}\left(\boldsymbol{\psi}_{\text {tilt }}^{T_{2}}\right)\right)\right. \\
\left.+\sum_{\forall s \in \mathcal{S}_{n}} \log _{2}\left(1+\hat{\gamma}_{s}^{b}\left(\boldsymbol{\psi}_{\text {tilt }}^{T_{n}}\right)\right)+\ldots+\sum_{\forall S \in \mathcal{S}_{N}} \log _{2}\left(1+\hat{\gamma}_{s}^{b}\left(\boldsymbol{\psi}_{\text {tilt }}^{T_{N}}\right)\right)\right\}
\end{aligned}
$$

where $N=|\mathcal{N}|$. According to (30) all the terms in the above series are in fact independent of each other, therefore the maximization can be performed on the individual terms of the series, so (31) can be written as:

$$
\begin{aligned}
\hat{\eta}_{\text {max }}=\max _{\boldsymbol{\psi}_{\text {tilt }}^{T_{1}}} \sum_{\forall s \in \mathcal{S}_{1}} \log _{2}\left(1+\hat{\gamma}_{s}^{b}\left(\boldsymbol{\psi}_{\text {tilt }}^{T_{1}}\right)\right)+\max _{\boldsymbol{\psi}_{\text {tilt }}^{T_{2}}} \sum_{\forall s \in \mathcal{S}_{2}} \log _{2}\left(1+\hat{\gamma}_{s}^{b}\left(\boldsymbol{\psi}_{\text {tilt }}^{T_{2}}\right)\right) \\
\max _{\boldsymbol{\psi}_{\text {tilt }}^{T_{n}}} \sum_{\forall s \in \mathcal{S}_{n}} \log _{2}\left(1+\hat{\gamma}_{s}^{b}\left(\boldsymbol{\psi}_{\text {tilt }}^{T_{n}}\right)\right)+\ldots+\max _{\boldsymbol{\psi}_{\text {tilt }}^{T_{N}}} \sum_{\forall s \in \mathcal{S}_{N}} \log _{2}\left(1+\hat{\gamma}_{s}^{b}\left(\boldsymbol{\psi}_{\text {tilt }}^{T_{N}}\right)\right)
\end{aligned}
$$

closing the summation gives following expression and thus proves the proposition

$$
\max _{\boldsymbol{\psi}_{\text {tilt }}^{B}} \hat{\zeta}=\sum_{\forall n \in \mathcal{N}}\left(\max _{\boldsymbol{\psi}_{\text {tilt }}^{T_{n}}} \sum_{\forall s \in \mathcal{S}_{n}} \log _{2}\left(1+\hat{\gamma}_{s}^{b}\right)\right)
$$

Referring back to the SO system of flocking birds, note that each bird adjusts its flight paramteres with reference to the observation of only two adjacent birds as a result though the group flight efficiency optimal formation i.e. V-shape is maintained only approximately, still significant group wide gain in flight efficiency is achieved. Similiary, here the $\operatorname{SIR} \hat{\gamma}_{s}^{b}\left(\boldsymbol{\psi}_{\text {tilt }}^{\hat{B}}\right)$ in (23) is based on interference perceived from adjacent two sectors only and therefore can achieve the objective in (21) only approximately (as shown through corollary 3) but significant system wide gain is possible as we will show in Section IV.

\section{E. Solving the Local Subproblem}

Based the arguments above, (27) can be solved by individually solving the $\mathrm{N}$ subproblems that appear in the summation in (33) as a small scale optimization problem over three tilt angles of the most interfering three adjacent cells only. This subproblem can be written as:

$$
\hat{\zeta}_{n, \max }=\max _{\psi_{t i l t}^{T_{n}}} \sum_{\forall s \in \mathcal{S}_{n}} w_{s} \log _{2}\left(1+\hat{\gamma}_{s}^{b}\right)
$$

Note that we have introduced a weight factor $w_{s}$ to be associated with each cell CG in the triplet. This weight factor can be used to model relative importance of each cell in a triplet, 
depending on the number of users in that cell, its size or its commercial significance and thus can capture certain aspects of the heterogeneity of the network at local scale.

The total achievable bandwidth normalized throughput at the CGs in a $n^{\text {th }}$ triplet is given as:

$$
\hat{\zeta}_{n}=w_{1} \log _{2}\left(1+\hat{\gamma}_{1}^{1}\right)+w_{2} \log _{2}\left(1+\hat{\gamma}_{2}^{2}\right)+w_{3} \log _{2}\left(1+\hat{\gamma}_{3}^{3}\right)
$$

where post scripts denote sector and subscripts denote CG's within given triplet as shown in figure 5. By substituting the value of $\hat{\gamma}_{s}^{b}$ from (23) in (35)

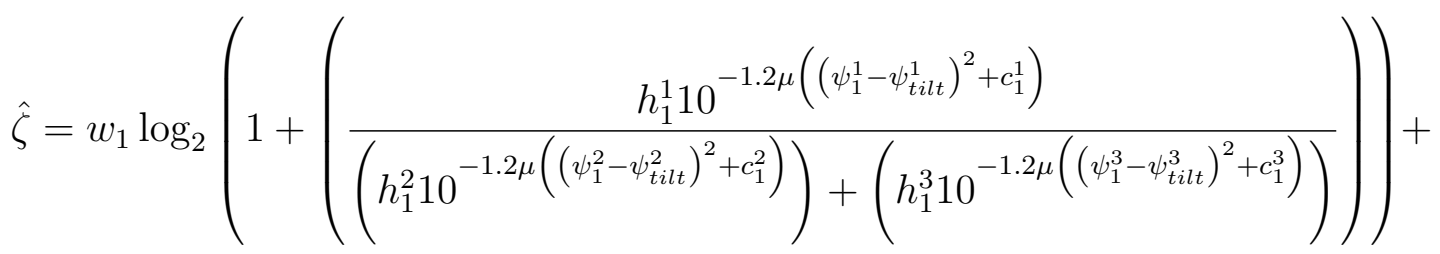

$$
\begin{aligned}
& w_{2} \log _{2}\left(1+\left(\frac{h_{2}^{2} 10^{-1.2 \mu}\left(\left(\psi_{2}^{2}-\psi_{\text {tilt }}^{2}\right)^{2}+c_{2}^{2}\right)}{\left(h_{2}^{1} 10^{-1.2 \mu\left(\left(\psi_{2}^{1}-\psi_{\text {tilt }}^{1}\right)^{2}+c_{2}^{1}\right)}\right)+\left(h_{2}^{3} 10^{-1.2 \mu\left(\left(\psi_{2}^{3}-\psi_{\text {tilt }}^{3}\right)^{2}+c_{2}^{3}\right)}\right)}\right)\right)+ \\
& w_{3} \log _{2}\left(1+\left(\frac{h_{3}^{3} 10^{-1.2 \mu}\left(\left(\psi_{3}^{3}-\psi_{\text {tilt }}^{2}\right)^{2}+c_{3}^{3}\right)}{\left(h_{3}^{1} 10^{-1.2 \mu\left(\left(\psi_{3}^{1}-\psi_{\text {tilt }}^{1}\right)^{2}+c_{3}^{1}\right)}\right)+\left(h_{3}^{2} 10^{-1.2 \mu}\left(\left(\psi_{3}^{2}-\psi_{\text {tilt }}^{2}\right)^{2}+c_{3}^{2}\right)\right.}\right)\right)
\end{aligned}
$$

We dropped the subscript $n$ to indicate that the analysis presented in sequel is valid for all triplets. The problem in (34) can be written in standard form:

$$
\max _{\psi_{\text {tilt }}^{1}, \psi_{\text {tilt }}^{2}, \psi_{\text {tilt }}^{3}} \hat{\zeta}\left(\psi_{\text {tilt }}^{1}, \psi_{\text {tilt }}^{2}, \psi_{\text {tilt }}^{3}\right)
$$

subject to:

$$
\psi_{\text {tilt }}^{1}, \psi_{\text {tilt }}^{2}, \psi_{\text {tilt }}^{3}<90^{\circ}
$$

As can be intuitively seen from the expanded form of the objective function of (37) in (36) and as we will observe in next section (37) is a non convex optimization problem. However, notice the fact that compared to (12), the problem in (37) is now a very a small scale and much simpler optimization problem, as number of optimization variables is only three compared to $|\mathcal{B}|$ and summation in optimization objective also has only three terms each with small constant evaluation complexity compared to $|\mathcal{R}|+|\mathcal{U} \backslash \mathcal{U}|$ terms in (12) each with evaluation complexity growing with $|\mathcal{B}|$. Note that the optimization parameters in (37) are confined to a finite range as $0^{\circ}<\psi<90^{\circ}$. Since, practically a tilt accuracy of up to $1^{\circ}$ is significant, the total search space of optimization problem in (37) is limited to maximum of $90 \times 90 \times 90=729000$. Given a reasonably 
small fixed search space, any of exhaustive search based heuristic can now used to quickly solve (37) with increased guarantee of quality of solution compared to large scale original problem. Or alternatively a solution can also be determined using a non linear optimization techniques that can tackle a small scale non convex optimization objective. For example, noticing that the objective function is twice differentiable and constraint is differentiable we can solve (37) using Sequential Quadratic Programming (SQP). To this end, the problem can be written in the standard form as:

$$
\min _{\psi}-\hat{\zeta}(\psi)
$$

subject to:

$$
g_{j}\left(\psi_{j}\right)<0 \quad, j=1,2,3
$$

where $\boldsymbol{\psi}=\left[\psi_{1}, \psi_{2}, \psi_{3}\right]$ and $g_{j}\left(\psi_{j}\right)=\psi_{j}-90$. Lagrangian of (38) can be given as:

$$
\mathcal{L}(\boldsymbol{\psi}, \boldsymbol{\lambda})=\hat{\zeta}(\boldsymbol{\psi})-\sum_{j=1}^{3} \lambda_{\mathbf{j}}\left(\psi_{j}-90\right)
$$

If $\hat{\mathbf{H}}$ denotes the approximate of the Hessian matrix $\mathbf{H}$, then we can define quadratic subproblem to be solved at $i^{\text {th }}$ iteration of SQP as follows:

$$
\min _{\boldsymbol{w} \in \Re^{J}} \frac{1}{2} \boldsymbol{w}^{T} \hat{\boldsymbol{H}}(\mathcal{L}(\boldsymbol{\psi}, \boldsymbol{\lambda}))_{i} \boldsymbol{w}+\nabla \hat{\zeta}(\boldsymbol{\psi})_{i} \boldsymbol{w}
$$

subject to:

$$
w_{j}+\psi_{j_{i}}-90<0 \quad j=1,2,3
$$

Below we briefly describe the three main steps to solve the above problem thorough SQP

1) Updating the $\hat{\boldsymbol{H}}$ : At each iteration the value of $\hat{\boldsymbol{H}}$ is updated using the Broyden-Fletcher -Goldfarb -Shanno (BFGS) approximation method i.e.

$$
\hat{\boldsymbol{H}}_{i+1}=\hat{\boldsymbol{H}}_{i}+\frac{\boldsymbol{b}_{i} \boldsymbol{b}_{i}^{T}}{\boldsymbol{b}_{i}^{T} \boldsymbol{a}_{i}}-\frac{\hat{\boldsymbol{H}}_{i}^{T} \boldsymbol{a}_{i}^{T} \boldsymbol{a}_{i} \hat{\boldsymbol{H}}_{i}}{\boldsymbol{a}_{i}^{T} \hat{\boldsymbol{H}}_{i} \boldsymbol{a}_{i}}
$$

where $\boldsymbol{a}_{i}=\boldsymbol{\psi}_{i+1}-\boldsymbol{\psi}_{i}$ and

$$
\boldsymbol{b}_{i}=\left(\nabla \hat{\zeta}(\boldsymbol{\psi})_{(i+1)}-\sum_{j=1}^{3} \lambda_{\mathbf{j} \nabla} g_{j,(i+1)}\right)-\left(\nabla \hat{\zeta}(\boldsymbol{\psi})_{(i)}-\sum_{j=1}^{3} \lambda_{\mathbf{j} \nabla} g_{j,(i)}\right)
$$

2) Solution of Quadratic subproblem: Once the Hessian is known the problem in (40) is a quadratic programming problem that can be solved using standard methods. We use gradient projection method as described in [37].

3) Line search and Merit function The solution of the quadratic subproblem in the $i^{\text {th }}$ iteration of SQP algorithm returns the vector $\boldsymbol{w}_{i}$ that provides the locus for next iteration as $\boldsymbol{\psi}_{i+1}=\boldsymbol{\psi}_{i}+\varrho \boldsymbol{w}_{\boldsymbol{i}}$ where $\varrho$ is set such that sufficient decrease in the merit function is achieved. we use merit function defined in [38] i.e. given as

$$
\varphi(\boldsymbol{\psi})=\hat{\zeta}(\boldsymbol{\psi})+\sum_{j=1}^{3} \mu_{j} . \max \left(0, g_{j}\left(\psi_{j}\right)\right)
$$


where $\mu$ is penalty parameters which we set as recommended in [38] i.e.

$$
\mu_{j,(i)}=\mu_{j,(i+1)}=\max _{j}\left\{\lambda_{j}, \frac{\mu_{j,(i)}+\lambda_{j}}{2}\right\}, \quad j=1,2,3
$$

Through the above steps of SQP, the problem in (37) can be solved within each triplet independently to determine the optimal tilt angles to be maintained by each of the three cells in the triplet for given locations of CG's within that triplet. The execution of these local solutions in each triplet in the cellular system locally results in achievement of the system wide goal in (21) approximately, that in turn manifests the original system-wide objective in (12) . Thus the optimal tilt angles can be maintained by dynamically responding to variations in cellular system environment, in distributed manner to maintain enhanced spectral efficiency on the BSRS links as well as on the BS-user links. In the following, we refer to this proposed solution as SOT (Self Organization of Tilts).

\section{Performance Evaluation}

In this section first we present the numerical results for SOT that are readily obtainable from the analysis presented above. This is followed by performance results of SOT evaluated by implementing SOT in a full scale system level simulator.

\section{A. Numerical Results}

1) Analysing Robustness of SOT: In this subsection we analyse sensitivity of SOT's gain to varying locations of $\mathrm{CG}$ and also to two key design parameter i.e. antenna height and vertical antenna beam width. The objective of the analysing SOT's gain sensitivity to these three factors is to investigate its robustness against randomness of user and BS relative locations, variety of BS heights and antenna types in real heterogeneous network. Numerical results for three random set of locations of CG's are plotted in figure 6. These results can be obtained by plotting (36) with $\beta=4, B_{v}=10^{\circ}, B_{h}=70^{\circ}$ and cell radius of $600 \mathrm{~m}$, BS and CG height of $20 \mathrm{~m}$ and $10 \mathrm{~m}$

respectively; and normalising $\hat{\zeta}$ by 3 i.e. the number of cells in the triplet. $\frac{\hat{\zeta}}{3}$ thus plotted in figure 6 gives the average spectral efficiency in a triplet. It can be seen that adaptation of antenna tilts can change the average spectral efficiency from 3.9 to $5.3,3.7$ to 4.7 and 2.1 to $2.8 \mathrm{~b} / \mathrm{s} / \mathrm{Hz}$ ( from top to bottom receptively), depending on the location of CGs that represent either RS or focal points of user distribution. Since SOT can dynamically determine the optimal tilt angles for any given locations of CG's in triplet, it can self-optimize antenna tilts to maintain maximum spectral efficiency. The values of spectral efficiency achieved by optimal tilts determined through SOT, 

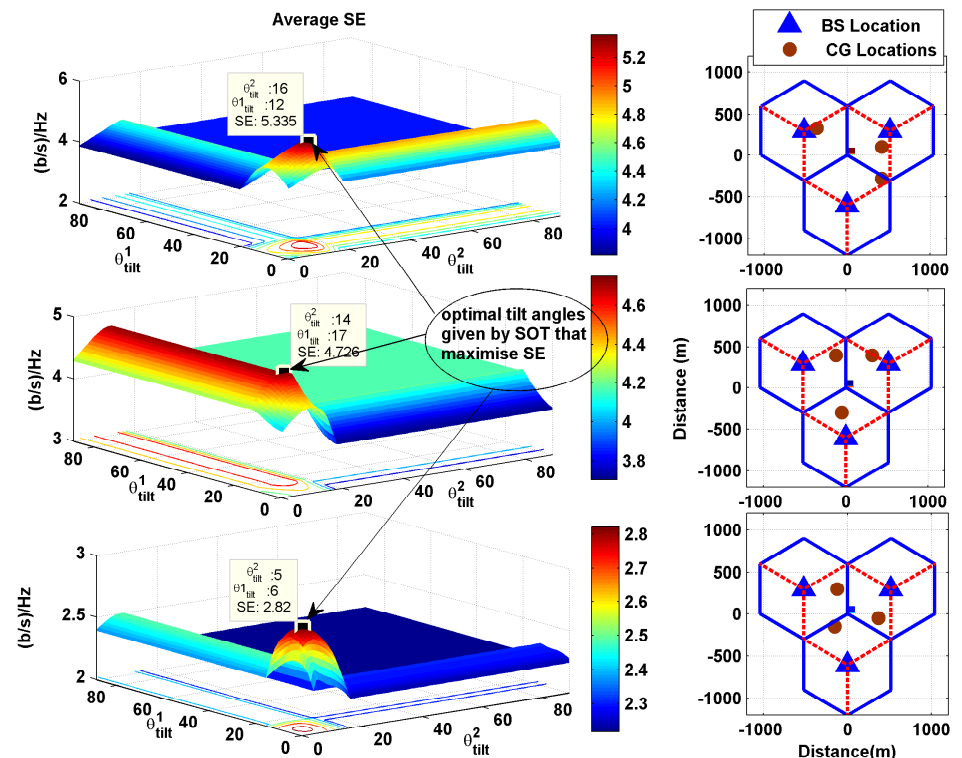

Fig. 6. $\frac{\hat{\zeta}}{3}$ plotted for a stand alone triplet against tilts of two sectors while third is fixed at $0^{\circ}$ for three different CG locations within the triplet. It can be seen that optimal tilt angles for maximum spectral efficiency change as locations of CGs change.

compared to the spectral efficiency achievable with the wide range of other tilts (see figure 6), imply that SOT, though the exact gain achievable by SOT is dependent on CG location and thus user distribution or RS location but in general it can yield a substantial gain in spectral efficiency compared to arbitrary tilting. It can be noticed from the contours plots in figure 6 that the optimal tilt angles generally lie in much smaller range e.g this range is just $0^{0}-20^{0}$ for the given cell radius and BS and RS heights. This observation can be used to further reduce the effective search space to only $20 \times 20 \times 20=8000$ combinations of tilt angles in a triplet to quickly determine the optimal tilt angles for any set of CG locations.

The gain SOT can yield, is also dependent on the vertical beamwidth of the antenna and the relative height of the BS compared to the height of the CG. Figure 7 plots the maximum spectral efficiency SOT yields for range of vertical beamwidth and the height of the BS above the height of $\mathrm{CG}$, for the locations of $\mathrm{CG}$ in the top right of figure 6 . Results show that, maximum achievable spectral efficiency by SOT, can be further increased as the height of the antenna increases or the vertical beamwidth decreases. A high antenna allows the front lobe of the antenna to be focused more precisely on the CGs as can be seen in figure 4. A narrow beamwidth on the other hand allows the antenna tilt to play a stronger role in boosting the desired signal and attenuating the interference. Thus a decrease in vertical beamwidth and increase in antenna height both result in higher SIR and thus higher spectral efficiency. 


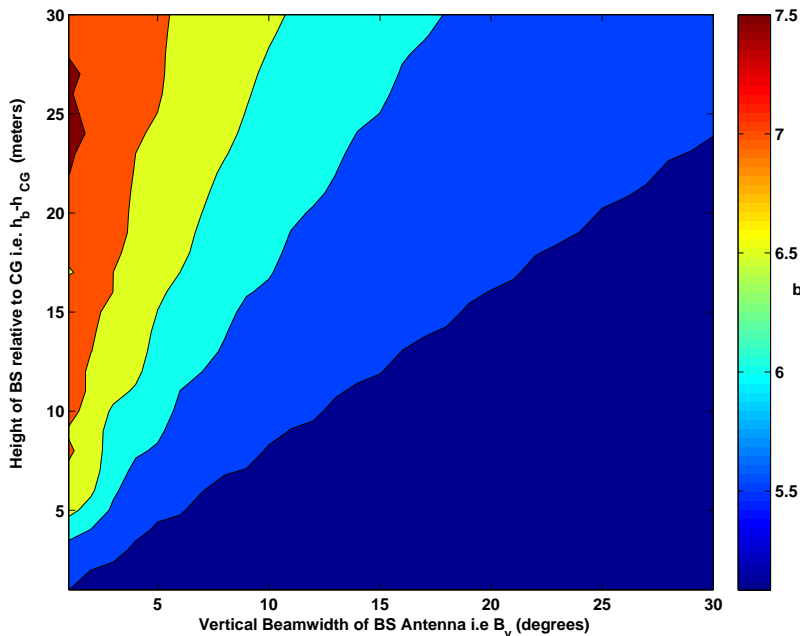

Fig. 7. Maximum spectral efficiency yielded by SOT for a given location CG's in triplet plotted for range of BS height above CG, and vertical beamwidth of antenna.

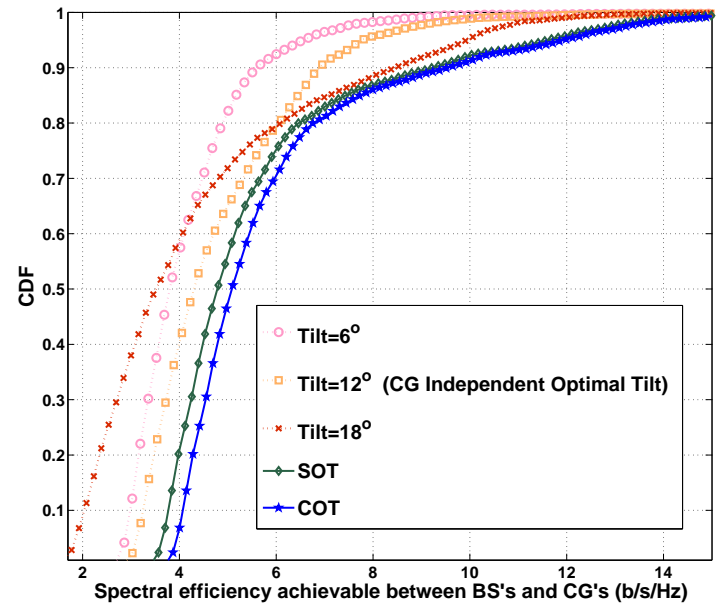

Fig. 8. SOT is compared with fixed tilting as well as Centralized Optimization of Tilts (COT). COT solution is obtained by solving (21) via brute force.

2) Comparing SOT with a Centralized Optimal Solution: While the numerical results above demonstrate that SOT can yield substantial gain for all possible CG locations and range of system design parameters, it is of interest to assess that how far this gain is from that achievable by an hypothetical optimal solution that can perform a Centralized Optimization of Tilts (COT). Unlike, SOT that has fixed search space of $90^{3}$ and thus can be solved easily, COT will require joint optimization of system-wide antenna tilts and thus will have search space of $90^{B}$.

The COT solution is obtained by solving (21) through brute force for $7 \times 3=21$ cells. Note that a cross-comparison with heuristic based solutions is omitted not only because of unavailability of an exact work in literature that considers relay enhanced cellular system, but also because the outputs of such solutions are largely dependent on the configuration of the underlying heuristics making a meaningful comparison difficult. On the contrary, chosen benchmarks are easily repeatable allowing fair cross-comparison and at the same time allow us to assess how much gain our solution yields compared current commercially used pragmatic solutions and how far is our distributed solution from a hypothetical system-wide centralized absolutely optimal SO solution. Due to the computational time constraint for COT, only tilt range of $6^{0}-18^{0}$ is considered with resolution of $2^{o}$. The rationale behind selecting this range is that it is centered around $12^{0}$. If consider the centroid of the cell to be the CG, which will be the case when user distribution is perfectly uniform as explained above, the fixed optimal tilt for given BS 
height of $32 \mathrm{~m}$, user height of $1.5 \mathrm{~m}$ and intersite distance of $500 \mathrm{~m}$, used (see Table I) is $12^{\circ}$, i.e. $\arctan \left((32-1.5) /\left(\frac{500}{2 \cos (30) 2}\right) \approx 12^{\circ}\right.$. Thus $6^{21}$ evaluations of objective function in (21) are traversed to find the optimal solution. On a regular desktop computer $(2.8 \mathrm{GHz}$ processor, $8 \mathrm{~GB}$ RAM) it took well over 8 hours. For fair comparison, SOT is also implemented under same set up of tilt range, resolution and the number of cells in the system.

Figure 8 plots CDF of spectral efficiency achievable on links assumed between CGs and BS, with SOT and COT. Note that albeit of relying on local information only, SOT's performance is considerably close to the COT. As expected, being globally optimal COT does outperforms SOT slightly. However, note that from real world implementation point of view COT is difficult to implement not only because of the tremendous computation effort required but also due the global signalling needed for its implementation (see Section V). The performance projected by COT in figure 8 does not take into account this large system wide signalling overheads. In terms of complexity, for even a cellular system as small as $19 \times 3$ cells (which is simulated for results in next subsection), the brute force based COT will have to do over $10^{99}$ evaluations of (21). Extrapolating the time of conducted experiment, that may take years. However, despite its impracticality COT does serve the purpose of an upper bound to bench mark our solution. The small gap that SOT has from COT, is worth of its distributed and system wide-signallingfree design that allows its computationally feasible solution and pragmatic implementation. In figure 8 , the CDFs with typical range fixed tilting values are also plotted for comparison with fixed range tilting that is often empirically set in commercial cellular systems. It can be noted that SOT outperforms all fixed tilting schemes including the fixed optimal tilt of $12^{0}$. Reasons for this gain provided by SOT are explained in next subsection.

\section{B. System Level Simulation Results}

The numerical results presented above show the gain of SOT for BS-CG links only, while considering interference from limited number of cells. As a real cellular system consists of large number of cells, containing randomly located RS and users of different heights and antenna gains, these factors will affect the system level performance of SOT. In order to evaluate the performance of SOT in more realistic scenarios, in this subsection we present results obtained by implementing SOT in a full scale system level simulator. Key modelling parameters used in system level performance evaluation are 3GPP compliant and are listed in Table I. Our system level simulator models an OFDMA based generic cellular system where half of cells contain 
TABLE I

3GPP Compliant System LeVel Simulation Parameters [39]

\begin{tabular}{|c|c|}
\hline Parameters & Values \\
\hline System topology & 19 BS with 3 sector/cells per BS \\
\hline BS Transmission Power & $46 \mathrm{dBm}$ \\
\hline BS Inter site distance & 500 meters \\
\hline BS height & 32 meters \\
\hline RS height & $5 \mathrm{~m}$ \\
\hline RS Type & Capacity Extension i.e. $w_{r}=1, \forall r \in R$ \\
\hline User height & 1.5 meters \\
\hline User activity levels & $a_{u}=1, \forall u \in U$ \\
\hline Network Topology Type & Homogenous, $w_{s}=1, \forall s \in S$ \\
\hline User antenna & $5 \mathrm{~dB}($ Omni directional) \\
\hline RS antenna & $7 \mathrm{~dB}$ (Omni directional) \\
\hline BS antenna horizontal beamwidth, $B_{h}$ & $70^{0}$ \\
\hline BS antenna vertical beamwidth, $B_{v}$ & $10^{\circ}$ \\
\hline BS antenna vertical Gain Weight,$\lambda_{v}$ & 0.5 \\
\hline BS antenna vertical Gain Weight , $\lambda_{h}$ & 0.5 \\
\hline BS antenna maximum gain, $G_{\max }$ & $14 \mathrm{~dB}$ \\
\hline BS antenna maximum attenuation, $A_{\max }$ & $25 \mathrm{~dB}$ \\
\hline Frequency & $2 \mathrm{GHz}$ \\
\hline Pathloss model & Urban, Scenario 1 [39] \\
\hline Shadowing standard deviation on BS-user links & $8 \mathrm{~dB}$ \\
\hline Shadowing standard deviation on BS-RS links & $4 \mathrm{~dB}$ \\
\hline
\end{tabular}

randomly located RS where other half selected randomly do not have RS and are served by BS only. Due to space limitations we present results for capacity enhancing RS only, as only in this case the backhaul optization becomes significant. To model capacity enhancing RS scenario, we assume that, in the cells with RS, $80 \%$ of the users in that cell are concentrated within $200 \mathrm{~m}$ radius from the RS. In cells without RS, users are randomly distributed across the cell. Simulator, is snapshot based and results reported are averaged over 10 snapshots of user and RS locations and tilt settings obtained via SOT for these user and RS distributions. Again comparison with prior works on heuristic based dynamic tilting schemes is omitted because of reasons explained above. Instead, for sake of reproducible performance evaluation, we compare the performance of SOT against range typical fixed antenna tilts including the fixed optimal tilt i.e. $0^{\circ}, 6^{\circ}, 12^{\circ}, 18^{\circ}$. Performance is evaluated for both BS-RS access links as well as BS-user coverage links.

Figure 9 plots the CDF of spectral efficiency achieved on the BS-RS access links. With Tilt $=0^{0}$ performance is worse obviously due to high interference. With a medium tilts of Tilt $=6^{\circ}, 12^{\circ}$ spectral efficiency improves as interference in general decreases for all BS-RS links. As the tilts are further increased i.e. Tilt $=18^{0}$ the spectral efficiency on the access links of RS that are located close to BS (50\%-tile and above) improves due to reduced interference and increased antenna gain focused to them, however the spectral efficiency on access links of RS located at the cell edges (around 5\%-tile and above) starts worsening, thereby nullifying the net gain in system wide average spectral efficiency. SOT, on the other hand provide a substantial net gain in spectral efficiency compared to all other fixed tilting options by dynamically setting 
tilts with respect to RS and CG locations.

Figure 10 plots the CDF of spectral efficiency achievable on BS-user links. The trends are same as observed for RS-BS links except that, in general the spectral efficiency on BS-user links is lower than that on access link. This is due to the different pathloss models on the two types of links i.e. BS-RS has much less shadowing than BS-user links. Furthermore, unlike RS that can be perfectly represented by one point in a cell used as CG in SOT, users are distributed all over the cells. Therefore, optimizing antenna tilts with respect to a single point that represent all users in cells ( i.e. CG), is though effective but not as much as it is for RS. For the same reason, high values of fixed tilts i.e. Tilt $=18^{0}$ has more adverse effect on BS-user links, than it has on BS-RS links as the large tilt can particularly cause outage for the cell edge users. The exact percentage of such outage may depend the antenna and transmission parameters and cell size. Nevertheless, it can been seen that SOT yields a net gain in spectral efficiency compared to fixed tilting on BS-user links as well, as it intelligently sets tilt values based on user concentrations.

A more quantitative perspective of the gain in spectral efficiency, SOT can give on BS-RS and BS-user links, is presented in figure 11 that plots the percentage gain in average spectral efficiency SOT yielded when compared to fixed optimal tilt of $12^{0}$ and no tilting at all. It can be observed that for in the cells with RS, BS-user links of the $20 \%$ user s that are not explicitly considered by SOT while deterging tilt, no significant gain is achieved compared to fixed optimal tilt as expected. However, for rest of the users, as well as RS that are considered in deterging the CGs yields very substantial gains compared to fixed tilting.

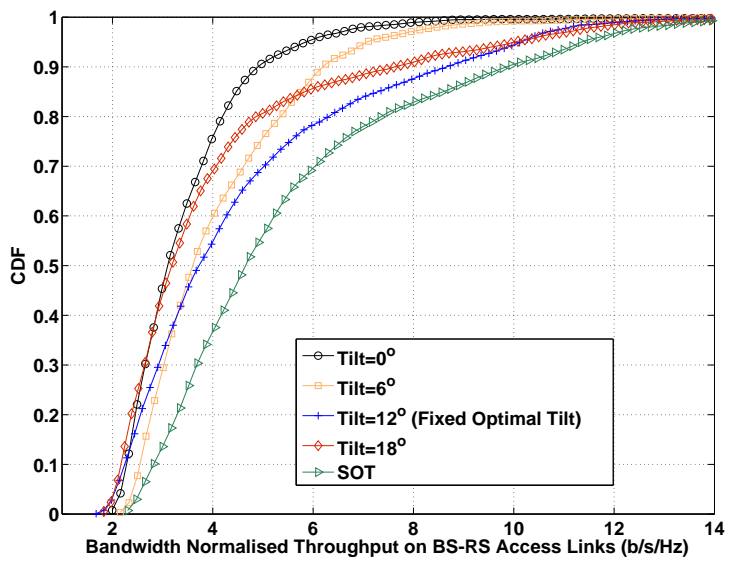

Fig. 9. CDF of spectral efficiency achievable on the BS-RS links with SOT and with classic fixed antenna tilts.

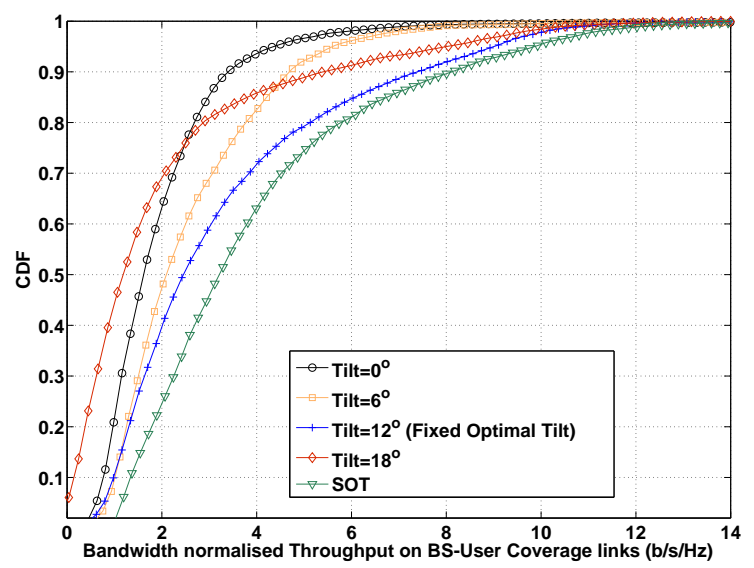

Fig. 10. CDF of spectral efficiency achievable on the BSusers links with SOT and with classic fixed antenna tilts. 


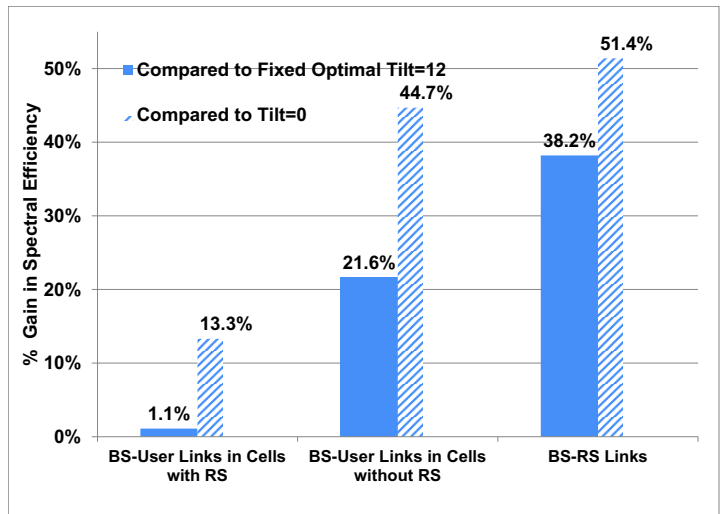

Fig. 11. Average spectral efficiency with SOT and with fixed tilts on BS-RS and BS-user links

\section{PRACTICAL IMPLEMENTATION OF SOT}

Although, in this paper we demonstrated the gain of proposed framework mainly in context of hexagonal grid model for tractability and brevity reasons, SOT is implementable in a real heterogeneous network as long as the network topology allows decomposition into local nonoverleaping cluster of cells (e.g. quartet, quintet, sextuplet) with same property as triplet i.e. a set of most interfering cells that can be repeated to cover whole network without overlap. The weight factors incorporated into the framework while calculating CGs can actually be used to take into account other types of heterogeneity such as cell sizes, sector spreads and azimuth angle biases, other than user profiling and RS types. Though exact gain of SOT will vary depending on actual system paramteres and topology as pointed out via results in section IV-A1, the key advantage of SOT is that it is practically implementable even with the state of the art technologies. Since the proposed framework does not incur heavy signaling overheads and has very low implementation complexity and cost, even reduced gain due to the irregularity of grid and propagation scattering is an added advantage compared to state of the art offline fixed empirical tilting. The RS and user positions information can be easily gathered at the respective BS with existing location estimation techniques such as GPS or the host of alternative cellular positioning techniques. For $\mathrm{RS}$, the location update will be required only when location of RS changes. So far need for user locations is concerned, as discussed earlier, as long as user distribution and activity level can be assumed to be uniform, the CG lies at the centroid of the sector can be determined by offline available system design parameters namely as cell radius, antenna pattern and height. 
Thanks to distributed nature of solution, even for the cells with highly dynamic non uniform user distribution, CG calculation requires SINR perceived by each user along with its position, to be known at the serving BS only, when a tilt update is needed. Since the SINR indicator is already available on BS in LTE in form of CQI (for scheduling purposes), this means negligible additional signalling is required to determine the CGs in each cell in online manner. The existing $\mathrm{X} 2$ interface can be used to promptly exchange the CG locations only, among the three adjacent cells that make each triplet. Based on this CG's information, the optimal tilt angle for all the three cells within each triplet in the system can be determined via SOT. Since, in emerging cellular systems, BS tilts can be adjusted electronically, thus with the implementation of SOT BS's can autonomously and dynamically maintain their antenna tilts to cope with changes in cellular eco system. Therefore, this algorithm requires no human intervention thereby promising significant OPEX saving. Another advantage of SOT is that due to its highly localized nature it is very agile as it does not suffer from excessive delays. Therefore, SOT can be implemented in an online manner using event based triggering mechanisms. Such triggering mechanisms can detect 'turning on or off 'of RSs or major variations in user demography, and thus can autonomously update the BS antenna tilts in the respective triplet(s) to maintain maximum spectral efficiency.

\section{CONCLUSIONS}

An analytical framework for distributed Self Organization of BS Tilts, named SOT, has been presented. SOT can autonomously determine and adapt optimal tilts in order to maximise spectral efficiency on the BS-RS as well as BS-user links in a live heterogeneous network, by taking into account users' and RSs' locations and activity levels. Both numerical and simulation results show that a gain of $10-50 \%$ in spectral efficiency compared to the typical fixed optimal tilting can be obtained with SOT depending on system topology and user demography. Comparison with a centralised tilt optimisation solution- which is difficult to implement in a real network due to its excessive signalling overhead and computational complexity- shows SOT can yield performance close to a centralized tilt optimisation solution. The key advantage of SOT is that it implementable with state of art technology and relies only on local signalling and thus features high scalability and agility. Therefore, it has potential for pragmatic implementation to autonomously optimise antenna tilts in a live cellular network in order to cope with either ever changing user demography or the impromptu deployment of new RSs. 


\section{ACKNOWLEDGMENT}

This work was made possible by NPRP grant No. 5 - 1047 - 2437 from the Qatar National Research Fund (a member of The Qatar Foundation).

\section{APPENDIX A}

In order to prove theorem 1 we need to show that:

$$
\sum_{\forall u \in \mathcal{U}_{b}} a_{u} \log _{2}\left(1+\tilde{\gamma}_{u}^{b}\left(\tilde{\psi}_{\text {tilt }}^{b}\right)\right)=\max _{\psi_{\text {tilt }}^{b}} \sum_{\forall u \in \mathcal{U}_{b}} a_{u} \log _{2}\left(1+\gamma_{u}^{b}\left(\psi_{\text {tilt }}^{b}\right)\right)
$$

if

$$
\int_{x} \int_{y} a_{(x, y)}\left(\left(\psi_{x, y}^{b}-\tilde{\psi}_{t i l t}^{b}\right) \frac{\tilde{\gamma}_{x, y}^{b}}{1+\tilde{\gamma}_{x, y}^{b}}\right) d x d y=0
$$

where $\mathcal{U}_{b}$ is set of user locations in $b^{\text {th }}$ cell such that $\mathcal{U}_{b} \subset \mathcal{U}$. Let $\tilde{\gamma}_{u}^{b}$ be the SIR in $b^{\text {th }}$ sector at $u^{\text {th }}$ point, with optimal antenna tilt given as:

$$
\tilde{\gamma}_{u}^{b}=\frac{d_{u}^{b-\beta} 10^{-1.2\left(\lambda_{v}\left(\frac{\psi_{u}^{b}-\tilde{\psi}_{t i l t}^{b}}{B_{v}}\right)^{2}+\lambda_{h}\left(\frac{\phi_{u}^{b}-\phi_{t i l t}^{b}}{B_{h}}\right)^{2}\right)}}{\sum_{\forall \hat{b} \in \mathcal{B} \backslash b}\left(d_{u}^{b^{-\beta}} 10^{-1.2\left(\lambda_{v}\left(\frac{\psi_{u}^{b}-\psi_{t i l t}^{b}}{B_{v}}\right)^{2}+\lambda_{h}\left(\frac{\phi_{u}^{b}-\phi_{t i l t}^{b}}{B_{h}}\right)^{2}\right)}\right)}
$$

Let $\tilde{\psi}_{t i l t}^{b}$ be the tilt that maximizes/minimizes the weighted sum throughput $\tilde{\zeta}^{b}$ in that cell, then

$$
\begin{aligned}
& \frac{\partial \tilde{\zeta}^{b}}{\partial \tilde{\psi}_{\text {tilt }}^{b}}=\frac{\partial}{\partial \tilde{\psi}_{\text {tilt }}^{b}}\left(\sum_{u=1}^{\left|\mathcal{U}_{b}\right|}\left(a_{u} \log _{2}\left(1+\tilde{\gamma}_{u}^{b}\left(\psi_{\text {tilt }}^{b}\right)\right)\right)\right)=0 \\
& \frac{1}{\ln 2} \sum_{u=1}^{\left|\mathcal{U}_{b}\right|} a_{u}\left(\frac{\frac{\partial}{\partial \tilde{\psi}_{t i l t}^{b}} \tilde{\gamma}_{u}^{b}\left(\psi_{\text {tilt }}^{b}\right)}{1+\tilde{\gamma}_{u}^{b}\left(\psi_{\text {tilt }}^{b}\right)}\right)=0 \\
& \frac{\partial \tilde{\gamma}_{u}^{b}}{\partial \tilde{\psi}_{t i l t}^{b}}=\frac{\frac{\partial}{\partial \tilde{\psi}_{\text {tilt }}^{b}} v}{\sum_{\forall \dot{b} \in \mathcal{B} \backslash b}\left(d_{u}^{b^{-\beta}} 10-1.2\left(\lambda_{v}\left(\frac{\psi_{u}^{b}-\psi_{t i l t}^{b}}{B_{v}}\right)^{2}+\lambda_{h}\left(\frac{\phi_{u}^{b}-\phi_{t i l t}^{b}}{B_{h}}\right)^{2}\right)\right)}
\end{aligned}
$$

$$
\begin{gathered}
\text { where } \\
\frac{\partial}{\partial \tilde{\psi}_{\text {tilt }}^{b}} v=\frac{\partial}{\partial \tilde{\psi}_{\text {tilt }}^{b}}\left(d_{u}^{b-\beta} 10-1.2\left(\lambda_{v}\left(\frac{\psi_{u}^{b}-\tilde{\psi}_{\text {tilt }}^{b}}{B_{v}}\right)^{2}+\lambda_{h}\left(\frac{\phi_{u}^{b}-\phi_{\text {tilt }}^{b}}{B_{h}}\right)^{2}\right)\right) \\
=d_{u}^{b-\beta} 1^{-1.2\left(\lambda_{v}\left(\frac{\psi_{u}^{b}-\tilde{\psi}_{\text {tilt }}^{b}}{B_{v}}\right)^{2}+\lambda_{h}\left(\frac{\phi_{u}^{b}-\tilde{\phi}_{\text {tilt }}^{b}}{B_{h}}\right)^{2}\right)} \ln 10 \frac{\partial}{\partial \tilde{\psi}_{\text {tilt }}^{b}}\left(-1.2\left(\lambda_{v}\left(\frac{\psi_{u}^{b}-\tilde{\psi}_{\text {tilt }}^{b}}{B_{v}}\right)^{2}+\lambda_{h}\left(\frac{\phi_{u}^{b}-\phi_{\text {tilt }}^{b}}{B_{h}}\right)^{2}\right)\right)
\end{gathered}
$$




$$
\begin{gathered}
=C\left(\psi_{u}^{b}-\tilde{\psi}_{t i l t}^{b}\right) d_{u}^{b}{ }^{-\beta} 10^{-1.2\left(\lambda_{v}\left(\frac{\psi_{u}^{b}-\tilde{\psi}_{t i l t}^{b}}{B_{v}}\right)^{2}+\lambda_{h}\left(\frac{\phi_{u}^{b}-\phi_{t i l t}^{b}}{B_{h}}\right)^{2}\right)} \\
C=\frac{2.4 \ln 10 \lambda_{v}}{B_{v}^{2}}
\end{gathered}
$$

Putting $v$ back in (50) and then using (50) in (49)

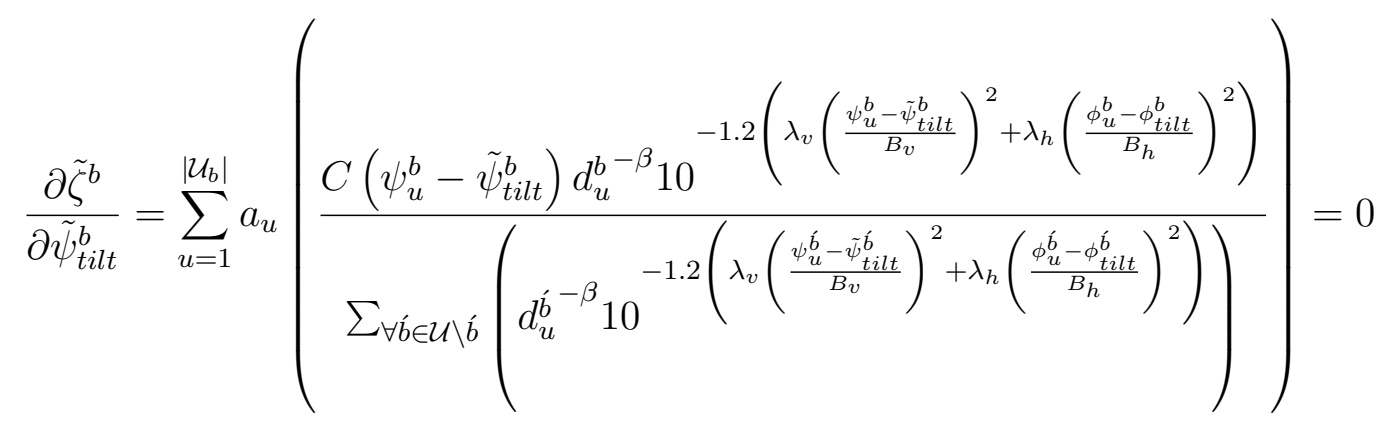

Using (47) in (55)

$$
\frac{\partial \tilde{\zeta}^{b}}{\partial \tilde{\psi}_{\text {tilt }}^{b}}=\sum_{u=1}^{\left|\mathcal{U}_{b}\right|} a_{u}\left(\left(\psi_{u}^{b}-\tilde{\psi}_{\text {tilt }}^{b}\right) \frac{\tilde{\gamma}_{u}^{b}}{1+\tilde{\gamma}_{u}^{b}}\right)=0
$$

It can be shown easily that $\frac{\partial \partial \tilde{\zeta}^{b}}{\partial \partial \tilde{\psi}_{\text {tilt }}^{b}}<0$, implying that stationary point at $\tilde{\psi}_{\text {tilt }}^{b}$ is a maximum. If user distribution is perfectly uniform and $u$ is substantially large, we can replace summation in (56) with the surface integral over whole area making it independent of individual user locations:

$$
\int_{x} \int_{y} a_{(x, y)}\left(\left(\psi_{x, y}^{b}-\tilde{\psi}_{t i l t}^{b}\right) \frac{\gamma_{x, y}^{b}}{1+\gamma_{x, y}^{b}}\right) d x d y=0
$$

where $a_{(x, y)}$ is weight associated with each point to reflect its importance. Hence theorem 1.

\section{REFERENCES}

[1] M. Dohler, R. Heath, A. Lozano, C. Papadias, and R. Valenzuela, "Is the phy layer dead?" Communications Magazine, IEEE, vol. 49, no. 4, pp. $159-165$, april 2011.

[2] A. Imran and R. Tafazolli, "Evaluation and comparison of capacities and costs of multihop cellular networks," 16th international conference on Telecommunications, pp. 160-165, 2009. [Online]. Available: http://portal.acm.org/citation.cfm?id=1700234.1700264

[3] K. Jacobson and W. Krzymien, "System design and throughput analysis for multihop relaying in cellular systems," IEEE Transactions on Vehicular Technology, vol. 58, no. 8, pp. 4514 -4528, Oct. 2009.

[4] 3GPP, "Self-organising networks; concepts and requirements," 3GPP TS 32.500 v10.1.0, Tech. Rep., 2010.

[5] S. Xu and Y. Hua, "Optimal design of spatial source-and-relay matrices for a non-regenerative two-way MIMO relay system,” IEEE Transactions on Wireless Communications, vol. 10, no. 5, pp. 1645 -1655, may 2011.

[6] O. Oyman, "Opportunistic scheduling and spectrum reuse in relay-based cellular networks," IEEE Transactions on Wireless Communications, vol. 9, no. 3, pp. 1074 -1085, march 2010.

[7] R. Babaee and N. Beaulieu, "Cross-layer design for multihop wireless relaying networks," IEEE Transactions on Wireless Communications, vol. 9, no. 11, pp. 3522 -3531, november 2010. 
[8] E. Amaldi, A. Capone, and F. Malucelli, "Optimizing UMTS radio coverage via base station configuration," in The 13th IEEE International Symposium on Personal, Indoor and Mobile Radio Communications, (PIMRC), vol. 1, sept. 2002, pp. $315-319$ vol.1.

[9] I. Siomina, P. Varbrand, and D. Yuan, "Automated optimization of service coverage and base station antenna configuration in UTMS networks," IEEE Wireless Communications, vol. 13, no. 6, pp. 16 -25, Dec. 2006.

[10] F. Gunnarsson, M. Johansson, A. Furuskar, M. Lundevall, A. Simonsson, C. Tidestav, and M. Blomgren, "Downtilted base station antennas - a simulation model proposal and impact on HSPA and LTE performance," in IEEE 68th Vehicular Technology Conference, (VTC Fall), Sept. 2008, pp. 1 -5.

[11] R. Pazhyannur, T. Dean, S. Anantha, and R. Dham, "RF optimization of WiMAX systems," in IEEE 68th Vehicular Technology Conference, (VTC Fall), sept. 2008, pp. 1 -5.

[12] O. N. Yilmaz, S. Hämälänen, and J. Hämäläinen, "Comparison of remote electrical and mechanical antenna downtilt performance for 3GPP LTE," in Proc. IEEE 70th Vehicular Technology Conf. 2009-Fall (VTC'09-Fall), 2009, pp. 1-5.

[13] R. Abou-Jaoude, N. Ulhaq, and C. Hartmann, "HSDPA throughput optimization with antenna tilt and pilot power in a moving hot-spot scenario," in IEEE 70th Vehicular Technology Conference,2009-Fall(VTC'09-Fall), 2009, pp. 1 -5.

[14] M. N. ul Islam, R. Abou-Jaoude, C. Hartmann, and A. Mitschele-Thiel, "Self-optimization of antenna tilt and pilot power for dedicated channels," in Proceedings of the 8th International Symposium on Modeling and Optimization in Mobile, Ad Hoc and Wireless Networks (WiOpt), May 2010, pp. 196 -203.

[15] R. Razavi, S. Klein, and H. Claussen, "Self-optimization of capacity and coverage in LTE networks using a fuzzy reinforcement learning approach," in Proc. IEEE 21st Int Personal Indoor and Mobile Radio Communications Symp,2010,(PIMRC'10), 2010, pp. 1865-1870.

[16] O. N. Yilmaz, J. Hämäläinen, and S. Hämälänen, "Self-optimization of remote electrical tilt," in IEEE 21st International Symposium on Personal Indoor and Mobile Radio Communications (PIMRC), sept. 2010, pp. 1128 -1132.

[17] _ _ "Optimization of adaptive antenna system parameters in self-organizing LTE networks," Wireless Networks, pp. 1-17, 2012. [Online]. Available: http://dx.doi.org/10.1007/s11276-012-0531-3

[18] M. Amirijoo, L. Jorguseski, R. Litjens, and R. Nascimento, "Effectiveness of cell outage compensation in LTE networks," in IEEE Consumer Communications and Networking Conference, Jan 2011, pp. 642 -647.

[19] H. Eckhardt, S. Klein, and M. Gruber, "Vertical antenna tilt optimization for LTE base stations," in IEEE 73rd Vehicular Technology Conference (VTC Spring), may 2011, pp. 1 -5.

[20] A. Awada, B. Wegmann, I. Viering, and A. Klein, "Optimizing the radio network parameters of the long term evolution system using taguchi's method," IEEE Transactions on Vehicular Technology, vol. 60, no. 8, pp. 3825 -3839, oct. 2011.

[21] H. Klessig, A. Fehske, G. Fettweis, and J. Voigt, "Improving coverage and load conditions through joint adaptation of antenna tilts and cell selection rules in mobile networks," in International Symposium on Wireless Communication Systems (ISWCS), aug. 2012, pp. $21-25$.

[22] N. Seifi, M. Coldrey, and M. Viberg, "Throughput optimization for miso interference channels via coordinated user-specific tilting," IEEE Communications Letters, vol. 16, no. 8, pp. 1248 -1251, august 2012.

[23] M. Naseer ul Islam and A. Mitschele-Thiel, "Cooperative fuzzy Q-Learning for self-organized coverage and capacity optimization," in IEEE 23rd International Symposium on Personal Indoor and Mobile Radio Communications (PIMRC), sept. 2012, pp. $1406-1411$.

[24] W. Luo, J. Zeng, X. Su, J. Li, and L. Xiao, "A mathematical model for joint optimization of coverage and capacity in self-organizing network in centralized manner," in 7th International ICST Conference on Communications and Networking 
in China (CHINACOM),, aug. 2012, pp. $622-626$.

[25] A. Thampi, D. Kaleshi, P. Randall, W. Featherstone, and S. Armour, "A sparse sampling algorithm for self-optimisation of coverage in LTE networks," in International Symposium on Wireless Communication Systems (ISWCS), aug. 2012, pp. $909-913$.

[26] S. Hurley, S. Allen, D. Ryan, and R. Taplin, "Modelling and planning fixed wireless networks," Wirel. Netw., vol. 16, no. 3, pp. 577-592, Apr. 2010. [Online]. Available: http://dx.doi.org/10.1007/s11276-008-0155-9

[27] O. Aliu, A. Imran, M. Imran, and B. Evans, "A survey of self organisation in future cellular networks," IEEE Communications Surveys Tutorials, vol. PP, no. 99, pp. 1 -26, 2012.

[28] R. Razavi, "Self-optimisation of antenna beam tilting in lte networks," in IEEE 75th Vehicular Technology Conference (VTC Spring), may 2012, pp. $1-5$.

[29] A. Imran, M. Imran, and R. Tafazolli, "Relay station access link spectral efficiency optimization through SO of macro BS tilts," IEEE Communications Letters, vol. 15, pp. 1326 - 1328, 2011.

[30] X. Yang and R. Tafazolli, "A method of generating cross-correlated shadowing for dynamic system-level simulators," in Personal, Indoor and Mobile Radio Communications, 2003. PIMRC 2003. 14th IEEE Proceedings on, vol. 1, sept. 2003, pp. 638 - 642 Vol.1.

[31] I. Viering, M. Dottling, and A. Lobinger, "A mathematical perspective of self-optimizing wireless networks," IEEE International Conference on Communications, 2009,(ICC '09), pp. 1 -6, June 2009.

[32] C. Prehofer and C. Bettstetter, "Self-organization in communication networks: principles and design paradigms," IEEE Communications Magazine, vol. 43, no. 7, pp. 78 - 85, july 2005.

[33] A. Imran, M. Bennis, and L. Giupponi, "Use of learning, game theory and optimization as biomimetic approaches for self-organization in macro-femtocell coexistence," in IEEE Wireless Communications and Networking Conference, april 2012, pp. $103-108$.

[34] T. Jansen, M. Amirijoo, U. Turke, L. Jorguseski, K. Zetterberg, R. Nascimento, L. Schmelz, J. Turk, and I. Balan, "Embedding multiple self-organisation functionalities in future radio access networks," in IEEE 69th Vehicular Technology Conference, (VTC Spring), 2009, pp. $1-5$.

[35] S. Hämäläinen, H. Sanneck, and C. Sartori, Eds., LTE Self-Organising Networks (SON): Network Management Automation for Operational Efficiency. Wiley, SBN 978-1-1199-7067-5, 2012.

[36] P. B. S. Lissaman and C. A. Shollenberger, "Formation flight of birds," Science, vol. 168, no. 3934, pp. 1003-1005, 1970. [Online]. Available: http://www.sciencemag.org/cgi/content/abstract/168/3934/1003

[37] P. Gill, W. Murray, and M. H. Wright, Practical Optimization. London, Academic Press, 1981.

[38] M. Powell, "A fast algorithm for nonlinearly constrained optimization calculations," in Numerical Analysis, ser. Lecture Notes in Mathematics, G. Watson, Ed. Springer Berlin / Heidelberg, 1978, vol. 630, pp. 144-157, 10.1007/BFb0067703. [Online]. Available: http://dx.doi.org/10.1007/BFb0067703

[39] A. B. Saleh, S. Redana, J. Hämäläinen, and B. Raaf, "On the coverage extension and capacity enhancement of inband relay deployments in lte-advanced networks," JECE, vol. 2010, pp. 4:1-4:10, Jan. 2010. [Online]. Available: http://dx.doi.org/10.1155/2010/894846 